\title{
Health-related quality of life in Chinese workers: a systematic review and meta- analysis
}

Ya Su ${ }^{1,2,3}$, Meng-Shuang Liu' ${ }^{1,2,3}$, Pinnaduwage Vijitha De Silva ${ }^{4}$, Truls Østbye ${ }^{5}$ and Ke-Zhi Jin ${ }^{1,2,3^{*}}$ (D)

\begin{abstract}
Background: Studies of health-related quality of life among workers have generated varying results. The purpose of this study was to conduct a systematic review to synthesize the scores of health-related quality of life measured by the World Health Organization Quality of Life questionnaire among Chinese workers and compare the results across gender, age, occupation and region.
\end{abstract}

Methods: Six databases including China National Knowledge Infrastructure, WanFang Data, China Science and Technology Journal Database, PubMed, Web of science and Scopus were searched for relevant publications in both English and Chinese from their inception to February 2021. Inclusion and exclusion criteria were established, and study and participant characters as well as health-related quality of life scores were extracted from included publications. Study quality was assessed by using the Crombie tool. The meta-analysis including individual publications used random-effects models. Subgroups analyses by gender, age, occupation and region were also conducted to explore the source of heterogeneity.

Results: One hundred thirty-nine out of 1437 potential publications were included. The pooled mean scores of healthrelated quality of life were 14.1 for the physical domain (95\%Cl: $13.9-14.3)$, 13.7 for the psychological domain (95\%Cl: $13.5-$ 13.8), 14.0 for the social relationship domain (95\%Cl: 13.8-14.2), 12.3 for the environment domain (95\%Cl: 12.1-12.5). No significant statistical difference was found between the different subgroups. Publication bias was present in the independence domain and the pooled scores were corrected to 15.0 (95\% Cl: 14.6-15.5) using the trim and fill method. Sensitivity analysis suggested that the results of the meta-analysis were stable. Region might be a source of heterogeneity. Workers in northeast China reported higher scores in the social relationship domain, and those in the central region reported lower scores in the environmental domain.

Conclusions: Chinese workers reported lower scores in four health-related quality of life domains than the general population. Region might be a potential influencing factor for workers' scores different, which needs further study. The pooled scores can served as benchmarks for workplace health promotion programes in Chinese workers and global occupational health studies.

Keywords: Health-related quality of life, Occupational health, Systematic review, Meta-analysis, China

\footnotetext{
* Correspondence: zhkjin@fudan.edu.cn

'Department of Occupational Health, School of Public Health, Fudan

University, 138 Yixueyuan Road Box288\#, Shanghai 200032, P.R. China

${ }^{2}$ Key Laboratory of Public Health Safety, Ministry of Education, Shanghai, P.R.

China

Full list of author information is available at the end of the article
}

(c) The Author(s). 2021, corrected publication 2021. Open Access This article is licensed under a Creative Commons Attribution 4.0 International License, which permits use, sharing, adaptation, distribution and reproduction in any medium or format, as long as you give appropriate credit to the original author(s) and the source, provide a link to the Creative Commons licence, and indicate if changes were made. The images or other third party material in this article are included in the article's Creative Commons licence, unless indicated otherwise in a credit line to the material. If material is not included in the article's Creative Commons licence and your intended use is not permitted by statutory regulation or exceeds the permitted use, you will need to obtain permission directly from the copyright holder. To view a copy of this licence, visit http://creativecommons.org/ licenses/by/4.0/. 


\section{Background}

Health-related quality of life (HRQOL) is defined by the World Health Organization (WHO) as "individuals' perceptions of their position in life in the context of the culture and value systems in which they live and in relation to their goals, expectations, standards and concerns" [1]. According to this definition, HRQOL is not only related to an individual's health status but also to their personal satisfaction. Therefore, HRQOL can vary greatly between China and other regions with different languages and culture. HRQOL instruments have been widely used in China since the 1980s. The Chinese versions of the World Health Organization Quality of Life (WHOQOL) questionnaire including WHOQOL-100 and WHOQOL-BREF were translated by Fang and his colleagues and were shown to have good reliability and validity in the Chinese population [2]. WHOQOL-BREF (26 items) is a simplified version based on WHOQOL100 (100 items). The items from the two scales were grouped into 4 domains: physical health, psychological health, social relationship and environment as well as evaluate general HRQOL and general health. The scores in each domain have good comparability between the two scales: the Pearson correlation coefficient ranges from 0.89 (the social relationship domain) to 0.95 (the physiological domain) [3]. WHOQOL-100 includes two additional domains: independence and spirituality beliefs.

Occupational activities run through most people's lives, and working conditions and environments have been recognized as important health determinants, i.e. key drivers of HRQOL. Different occupational groups may experience various and different health problems due to the nature of their jobs, with different performance in HRQOL. For example, high physical work demand and awkward static/repetitive working postures may contribute to higher incidence of musculoskeletal disorders $[4,5]$; shift work is related to cardiovascular heart disease and mental disorders [6, 7]; and sedentary behavior is a risk factor for chronic diseases including obesity, diabetes, etc. In addition, male and female workers at different ages may have different types of job, e.g. nurses and teachers are mostly women, while bluecollar workers (such as construction workers and miners) are mostly young men. Therefore, it is important to assess HRQOL by gender, age and occupation to identify differences and group time trends with a view to providing group specific occupational health services. The influence of different geographical regions on the results also needs to be explored, taking into account differences in climate, lifestyle and subtle cultural differences.

Although individual studies have reported results based on WHOQOL in Chinese workers engaged in different occupations, there has been no other systematic review summarizing these findings. Therefore, the primary objective of this systematic review was to summarize the findings around six HRQOL domains in Chinese workers, so as to provide references for future studies and for health policy (Studies using either of the two versions of the questionnaires generated the scores for physical health, psychological health, social relationship and environment domain, while only those using WHOQOL-100 generated the scores for independence and spirituality beliefs domain). The second objective was to compare the results across gender, age groups and occupational groups in order to explore the characteristics of different subgroups and identify more vulnerable groups.

\section{Methods}

The protocol for this systematic review with metaanalysis was registered in the International Prospective Register of Systematic Reviews (PROSPERO, Registration ID: CRD42020151775). The current review was reported by following the guidelines of Preferred Reporting Items for Systematic Reviews and MetaAnalysis (PRISMA) Statement [8]. Two reviewers (SU and LIU) independently searched and selected the publications. Any disagreement led to a consultation with the third reviewer (JIN) and resolved by reaching consensus.

\section{Data sources and search strategy}

Potential publications were identified from six databases searched from their inception and up to February 2021: China National Knowledge Infrastructure (CNKI), WanFang Data (WF), China Science and Technology Journal Database (CQVIP), PubMed, Web of Science and Scopus. Of these databases, CNKI, WF and CQVIP mainly covered Chinese publications. Keywords, medical subject heading $(\mathrm{MeSH})$ terms and free-text words were used as searching strings. The search strategy incorporated two principal components. The first related to the study population: Chinese workers with active employment and engaged in any specific industries. The second related to the health outcome, namely HRQOL evaluated by WHOQOL-BREF or WHOQOL-100. The exact search strategies are presented in Table 1.

\section{Study eligibility}

The inclusion criteria were as follows: (1) cross-sectional study, or cohort, intervention study reporting baseline data; (2) conducted in the Chinese mainland; (3) active occupational population with specific occupation; (4) HRQOL measured using WHOQOL-BREF or WHOQOL-100; (5) publications in Chinese or English until February, 2021. Publications were excluded if they: 
Table 1 Search strategies in CNKI, WF, CQVIP, PubMed, Web of Science and Scopus

\begin{tabular}{|c|c|c|c|}
\hline Database & Nation & Occupation group & Quality of life \\
\hline CNKI & - & 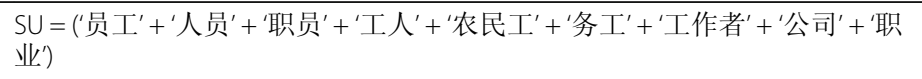 & $\begin{array}{l}\text { TKA = ('世界卫生组织生存质 } \\
\text { 量' }+ \text { 'WHOQOL') }\end{array}$ \\
\hline WF & - & $\begin{array}{l}\text { 主题:(“员工” OR “人员” OR “职员” OR “工人” OR “农民工” OR “务工” OR “工作 } \\
\text { 者” OR “公司” OR “职业”) }\end{array}$ & $\begin{array}{l}\text { 摘要:(“世界卫生组织生存质量” } \\
\text { OR “WHOQOL") }\end{array}$ \\
\hline CQVIP & - & $\begin{array}{l}M=\text { (员工 } O R \text { 人员 } O R \text { 职员 } O R \text { 工人 } O R \text { 农民工 } O R \text { 务工 } O R \text { 工作者 } O R \text { 公司 } \\
\text { OR 职业) }\end{array}$ & $\begin{array}{l}\mathrm{R}=\text { (世界卫生组织生存质量 OR } \\
\text { WHOQOL) }\end{array}$ \\
\hline PubMed & $\begin{array}{l}\text { (China [ALL] OR } \\
\text { Chinese [ALL]) }\end{array}$ & $\begin{array}{l}\text { (workplace[MH] OR occupations[MH] OR occupational groups[MH] OR work[MH] } \\
\text { OR employ*[ALL] OR workplace[ALL] OR workplaces[ALL] OR occupation*[ALL] } \\
\text { OR work*[ALL] OR profession*[ALL] OR labor[ALL] OR labour[ALL] OR job[ALL] } \\
\text { OR jobs[ALL] OR personnel[ALL] OR personnels[ALL] OR staff[ALL] OR staffs[ALL] } \\
\text { OR "green collar"[ALL] OR "pink collar"[ALL] OR "white collar"[ALL] OR "blue } \\
\text { collar"[ALL] OR company[ALL] OR companies[ALL] OR corporation[ALL] OR } \\
\text { corporations[ALL] OR enterprise[ALL] OR enterprises[ALL]) }\end{array}$ & $\begin{array}{l}\text { ("world health organization quality } \\
\text { of life"[ALL] OR WHOQOL[ALL]) }\end{array}$ \\
\hline $\begin{array}{l}\text { Web of } \\
\text { Science }\end{array}$ & $\begin{array}{l}\text { TS }=(\text { China OR } \\
\text { Chinese) }\end{array}$ & $\begin{array}{l}\text { TS = (employ* OR workplace\$ OR occupation* OR work* OR profession* OR } \\
\text { labo\$r OR job\$ OR personnel\$ OR staff\$ OR "green collar" OR "pink collar" OR } \\
\text { "white collar" OR "blue collar" OR company OR companies OR corporation\$ OR } \\
\text { enterprise\$) }\end{array}$ & $\begin{array}{l}\text { TS = ("the world health } \\
\text { organization quality of life" OR } \\
\text { WHOQOL) }\end{array}$ \\
\hline Scopus & $\begin{array}{l}\text { TITLE-ABS-KEY } \\
\text { (China OR } \\
\text { Chinese) }\end{array}$ & $\begin{array}{l}\text { TITLE-ABS-KEY (employ* OR workplace OR workplaces OR occupation* OR work* } \\
\text { OR profession* OR labor OR labour OR job OR jobs OR personnel OR personnels } \\
\text { OR staff OR staffs OR "green collar" OR "pink collar" OR "white collar" OR "blue } \\
\text { collar" OR company OR companies OR corporation OR corporations OR } \\
\text { enterprise OR enterprises) }\end{array}$ & $\begin{array}{l}\text { ALL ("the world health organization } \\
\text { quality of life" OR whoqol) }\end{array}$ \\
\hline
\end{tabular}

(1) did not report specific scores or standard deviations;

(2) reported nonstandard data (incomparability data that were not calculated according to standard methods); (3) included workers with specific diseases; (4) repeated findings from other analyses that measured the same population at the same study period; (5) were special groups providing goods or services prohibited by local law (e.g. sex workers).

\section{Data extraction}

The extracted data from the included publications contained: (1) study characteristics (author, published year, etc.); (2) participant characteristics (age, gender, occupation, region, response rates etc.); (3) health outcomes (sample sizes, average scores and standard deviation for different domain of HRQOL). Microsoft Excel 2016 was used for data management.

\section{Quality assessment}

The methodological quality of each study was evaluated using a well-established quality appraisal tool recommended by Crombie [9]. The tool and its modified version have been used in many systematic reviews [1012]. The tool consists of 7 items with responses "Yes ( 1 point)" or "No (0 point)". Consequently, each study provided a score between 0 and 7 . The scores were grouped into: $\leq 5$ (low quality) and $>5$ (high quality).

\section{Statistical analysis}

To ensure comparability of data, scores on a $0-100$ scale were transformed to a $0-20$ scale following a procedure stated by the WHOQOL User Manual [13]. A meta- analysis was conducted for each domain of HRQOL to estimate the combined means and 95\% confidence intervals. The test for heterogeneity among results and the selection of random effects model or fixed effects model were determined according to the I-squared statistics. Publication bias was assessed by Funnel plot, Egger's test and Begg's test. A $p$-value $<0.05$ was considered statistically significant publication bias. The trim and fill method was further used to assess the influence of bias on the results. Influence analysis was conducted with each study deleted from the model to explore the stability of the results in the meta-analysis. Stata 15 was used for statistical analysis.

\section{Results \\ Selection process}

Figure 1 shows the flow diagram describing the study and publication selection process. A total of 1437 publications were initially identified from the databases or the related references, 1026 remained after removing duplicates electronically or manually. Next, 795 and 92 publications were removed by screening titles/abstracts and full text according to inclusion and exclusion criteria. Of the excluded full-text publications, 34 publications were found reported duplicated findings, and 11 publications were found reporting nonstandard data. The remaining 139 remained for quantitative synthesis.

\section{Publication characteristics and study quality}

Table 2 describes the study and participant characteristics of each publication. The included publications reported a total of 98,144 workers engaged in 


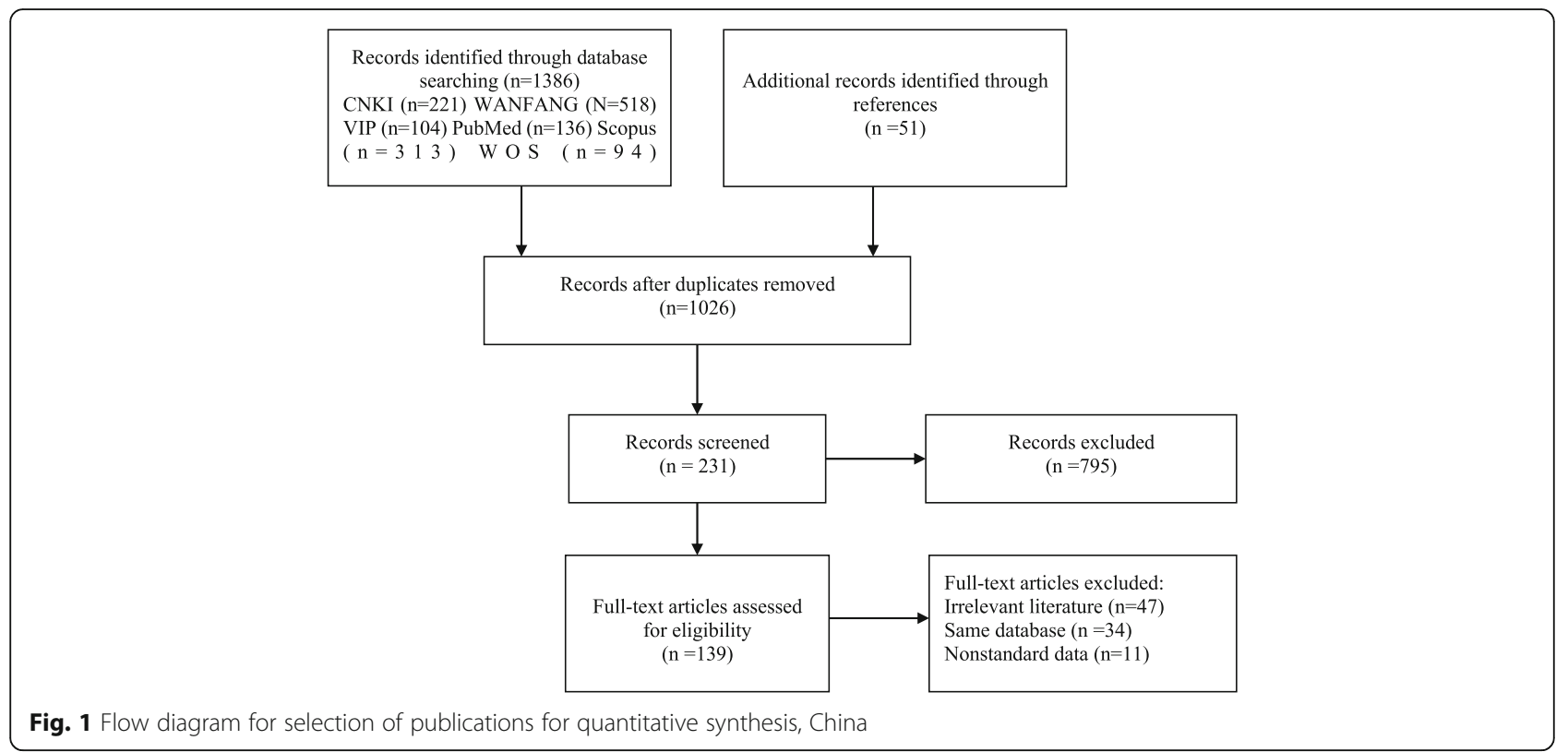

construction, manufacturing, natural resource extraction, education, health and other working fields. Thirty-four publications only reported the numbers of participants in different age groups, so the mean age was estimated according to the mid-value of each age group. The mean age reported varied from 19.8 to 66.5. Female workers dominated the education and health care workforce, while male workers dominated in the military, mining, construction and manufacturing industries. The sample size reported in included publications ranged from 40 to 25,066, 60.4\% $(n=84)$ had more than 300 participants. Twenty-one publications used the WHOQOL-100 questionnaire, whlie the remaining used the WHOQOL-BREF.

The study quality assessment of those publications can also be seen in Table 2. The average score was 4.7, ranging from 3 to $7.74 .8 \%(n=104)$ of publications were rated as having low study quality. The variation in scores mainly reflected in the items "appropriateness of design to meet the aims" and "clearly stated aims and likelihood of reliable and valid measurements". Only 9 publications explicitly stated that random sampling or the whole population was used, and only $54.7 \%(n=76)$ reported reliability or validity of the questionnaires used in the investigation.

\section{Meta-analysis}

The scores in the physical $(n=138)$, psychological $(n=138)$, social relationship $(n=137)$, environment $(n=136)$, independence $(n=23)$ and spirituality beliefs $(n=21)$ domains varied from 10.9-18.0, 11.1-16.6, $10.0-18.1,10.0-19.2,12.1-16.7$, and $10.8-14.7$, respectively. The heterogeneity test showed significant differences among the results of included publications, $\mathrm{I}^{2}>98 \%, \quad P<0.001$. Therefore, the random effects model was used for data synthesis. The estimated mean scores were 14.1 for the physical domain (95\%CI: 13.9-14.3), 13.7 for the psychological domain (95\%CI: $13.5-13.8), 14.0$ for the social relationship domain (95\%CI: 13.8-14.2), 12.3 for the environment domain (95\%CI: 12.1-12.5), 15.3 for the independence domain (95\%CI: 14.8-15.8), and 11.8 for the spirituality beliefs domain (95\%CI: 11.30-12.3). Besides, 26 publications reported the general HRQOL and 21 reported general health, and the pooled scores were 3.3 (95\%CI: 3.2-3.5), 3.2(3.2-3.5). The forest plots are shown in Fig. 2.

The analysis included publications from 2001 to 2021. HRQOL scores in the six domains each year were similar and showed no trend over time $(P>0.05)$. The maximum differences in the mean score for the physical, psychological, social relationship, and environment domain from year to year were 1.8, 1.4, 1.1, and 2.3, respectively.

\section{Publication bias assessment and sensitivity analysis}

Visual inspection of the funnel plot (Fig. 3), Egger's test and Begg's test did not suggest publication bias in the meta-analysis of the physical, psychological, social relationship environment and spirituality beliefs domains $(P>0.05)$. However, Egger's test suggested potential publication bias in the independence domain $(P=0.011)$, while Begg's test did not $(P=0.853)$. Therefore, the trim and fill method was also applied, and it indicated that if 4 estimated missing publications were added, then the pooled score of the independence domain would change 
Table 2 Descriptive characteristics and quality assessment of the included publications

\begin{tabular}{|c|c|c|c|c|c|c|c|}
\hline Author(year) & Occupation & $\begin{array}{l}\text { Age (mean } \pm \\
\text { standard } \\
\text { deviation, } \\
\text { range) }\end{array}$ & $\begin{array}{l}\text { Gender } \\
\text { (\%male) }\end{array}$ & $\begin{array}{l}\text { Sample size } \\
\text { (effective } \\
\text { response } \\
\text { rate) }\end{array}$ & Questionnaire & $\begin{array}{l}\text { Region of } \\
\text { work } \\
\text { (province) }\end{array}$ & $\begin{array}{l}\text { Quality } \\
\text { assessment } \\
\text { score }\end{array}$ \\
\hline $\begin{array}{l}\text { Huang et al. } \\
\text { (2001) [14] }\end{array}$ & Nurses & $\begin{array}{l}31.2 \pm 8.9,18- \\
55\end{array}$ & $0 \%$ & $522(94.9 \%)$ & 100 & Hubei & 5 \\
\hline $\begin{array}{l}\text { Liu et al. } \\
\text { (2004) [15] }\end{array}$ & Medical staff & $33.7 \pm 9.1$ & $37.3 \%$ & 807 (89.7\%) & 100 & Hunan & 6 \\
\hline $\begin{array}{l}\text { Wang et al. } \\
\text { (2005) [16] }\end{array}$ & Military personnel & $\begin{array}{l}21.6 \pm 3.7,16- \\
44\end{array}$ & $100 \%$ & $612(96.5 \%)$ & BREF & $\begin{array}{l}\text { Inner } \\
\text { Mongolia }\end{array}$ & 6 \\
\hline $\begin{array}{l}\text { Chen et al. } \\
\text { (2005) [17] }\end{array}$ & Nurses & $\begin{array}{l}33.3 \pm 8.7,18- \\
56\end{array}$ & $0 \%$ & $1053(90.0 \%)$ & 100 & Jiangsu & 4 \\
\hline $\begin{array}{l}\text { Li et al. } \\
\text { (2005) [18] }\end{array}$ & Military convalescents & $\begin{array}{l}66.5 \pm 9.7,37- \\
85\end{array}$ & $86.5 \%$ & $\begin{array}{l}244 \\
\text { (Unknown) }\end{array}$ & BREF & Guangdong & 3 \\
\hline $\begin{array}{l}\text { Jing et al. } \\
\text { (2005) [19] }\end{array}$ & Oculists & $33.3 \pm 9.3^{\mathrm{a}}$ & $32.2 \%$ & $311(94.2 \%)$ & BREF & Guangdong & 6 \\
\hline $\begin{array}{l}\text { Zhao et al. } \\
\text { (2006) [20] }\end{array}$ & Military personnel & $21.3 \pm 3.0$ & $100 \%$ & 485 (99.0\%) & BREF & Tibet & 5 \\
\hline $\begin{array}{l}\text { Geng et al. } \\
\text { (2006) [21] }\end{array}$ & Armed polices & $\begin{array}{l}21.2 \pm 3.1,17- \\
33\end{array}$ & $100 \%$ & $1283(100 \%)$ & BREF & Guangdong & 4 \\
\hline $\begin{array}{l}\text { Tang et al. } \\
\text { (2006) [22] }\end{array}$ & Military personnel & $\begin{array}{l}20.8 \pm 2.3,17- \\
33\end{array}$ & $100 \%$ & $\begin{array}{l}215 \\
\text { (Unknown) }\end{array}$ & BREF & Unknown & 4 \\
\hline $\begin{array}{l}\text { Tang et al. } \\
\text { (2006) [23] }\end{array}$ & Hospital temporary workers & Unknown & Unknown & $562(93.7 \%)$ & 100 & Shenzhen & 4 \\
\hline $\begin{array}{l}\text { Yang et al. } \\
\text { (2006) [24] }\end{array}$ & Middle school teachers & Unknown & $18.4 \%$ & $718(89.4 \%)$ & BREF & Hebei & 5 \\
\hline $\begin{array}{l}\text { Liu et al. } \\
\text { (2007) [25] }\end{array}$ & Nurses & $29.9 \pm 8.6^{a}$ & Unknown & $96(96.0 \%)$ & 100 & Heilongjiang & 3 \\
\hline $\begin{array}{l}\text { Liu et al. } \\
\text { (2007) [26] }\end{array}$ & Roadmen & $29.8 \pm 9.1$ & $100 \%$ & $\begin{array}{l}376 \\
\text { (Unknown) }\end{array}$ & BREF & Hubei & 4 \\
\hline $\begin{array}{l}\text { Chen et al. } \\
\text { (2007) [27] }\end{array}$ & Nurses & $34.8 \pm 9.2$ & Unknown & 1648 (92.7\%) & BREF & Shandong & 4 \\
\hline $\begin{array}{l}\text { Zhou et al. } \\
\text { (2007) [28] }\end{array}$ & Middle SchoolTeachers & $\begin{array}{l}36.2 \pm 8.0,19- \\
60\end{array}$ & $45.5 \%$ & $622(95.7 \%)$ & BREF & Hunan & 6 \\
\hline $\begin{array}{l}\text { Liu et al. } \\
\text { (2007) [29] }\end{array}$ & Armed police forces & $19.8 \pm 1.9$ & $100 \%$ & $516(97.4 \%)$ & BREF & Qinghai & 6 \\
\hline $\begin{array}{l}\text { Yang et al. } \\
\text { (2008) [30] }\end{array}$ & Scientific research personnel & $22-85$ & $32.4 \%$ & $272(95.4 \%)$ & 100 & Beijing & 5 \\
\hline $\begin{array}{l}\text { Wang et al. } \\
\text { (2008) [31] }\end{array}$ & Nurses & $\begin{array}{l}31.5 \pm 4.9,21- \\
44\end{array}$ & $0 \%$ & $189(94.5 \%)$ & BREF & Guangdong & 4 \\
\hline $\begin{array}{l}\text { Tang et al. } \\
\text { (2008) [32] }\end{array}$ & Military personnel & Unknown & Unknown & $2581(92.2 \%)$ & BREF & Unknown & 5 \\
\hline $\begin{array}{l}\text { Tang et al. } \\
\text { (2008) [33] }\end{array}$ & Nurses & $\begin{array}{l}32.5 \pm 8.5,18- \\
53\end{array}$ & $0 \%$ & $574(94.7 \%)$ & 100 & Guangdong & 6 \\
\hline $\begin{array}{l}\text { Du et al. } \\
\text { (2008) [34] }\end{array}$ & Gym coaches & $27.0 \pm 5.6^{a}$ & $64.9 \%$ & $97(75.8 \%)$ & BREF & $\begin{array}{l}\text { Shanghai, } \\
\text { Jiangsu }\end{array}$ & 5 \\
\hline $\begin{array}{l}\text { Liu et al. } \\
\text { (2008) [35] }\end{array}$ & Nurses & $36.0,18-60$ & $0 \%$ & $479(95.8 \%)$ & BREF & Shandong & 5 \\
\hline $\begin{array}{l}\text { Yu et al. } \\
\text { (2008) [36] }\end{array}$ & Coal workers & $19-50$ & $56.2 \%$ & $505(93.5 \%)$ & BREF & Shanxi & 7 \\
\hline $\begin{array}{l}\text { Zhang et al. } \\
\text { (2008) [37] }\end{array}$ & Furniture maker & $\begin{array}{l}29.5 \pm 8.6,17- \\
52\end{array}$ & $83.5 \%$ & 85 (Unknown) & BREF & Beijing & 5 \\
\hline $\begin{array}{l}\text { Su et al. } \\
\text { (2008) [38] }\end{array}$ & Middle SchoolTeachers & $\begin{array}{l}33.6 \pm 7.5,21- \\
57\end{array}$ & $34.7 \%$ & $759(94.9 \%)$ & 100 & Shandong & 6 \\
\hline Dong et al. & Nurses & $34.7 \pm 8.3$ & Unknown & $115(76.7 \%)$ & 100 & Yunnan & 3 \\
\hline
\end{tabular}


Table 2 Descriptive characteristics and quality assessment of the included publications (Continued)

\begin{tabular}{|c|c|c|c|c|c|c|c|}
\hline Author(year) & Occupation & $\begin{array}{l}\text { Age (mean } \pm \\
\text { standard } \\
\text { deviation, } \\
\text { range) }\end{array}$ & $\begin{array}{l}\text { Gender } \\
\text { (\%male) }\end{array}$ & $\begin{array}{l}\text { Sample size } \\
\text { (effective } \\
\text { response } \\
\text { rate) }\end{array}$ & Questionnaire & $\begin{array}{l}\text { Region of } \\
\text { work } \\
\text { (province) }\end{array}$ & $\begin{array}{l}\text { Quality } \\
\text { assessment } \\
\text { score }\end{array}$ \\
\hline \multicolumn{8}{|l|}{ (2008) [39] } \\
\hline $\begin{array}{l}\text { Li et al. } \\
\text { (2008) [40] }\end{array}$ & Doctors & $39.7 \pm 8.3$ & $63.5 \%$ & $200(80.0 \%)$ & 100 & Chongqing & 4 \\
\hline $\begin{array}{l}\text { Liu et al. } \\
\text { (2009) [41] }\end{array}$ & Reconstruction personnel after earthquake & $39.5 \pm 6.0$ & $96.4 \%$ & $\begin{array}{l}112 \\
\text { (Unknown) }\end{array}$ & BREF & Sichuan & 3 \\
\hline $\begin{array}{l}\text { Tang et al. } \\
\text { (2009) [42] }\end{array}$ & Military personnel & $\begin{array}{l}22.8 \pm 3.8,16- \\
48\end{array}$ & $99.8 \%$ & $2305(95.8 \%)$ & BREF & $\begin{array}{l}\text { Shanghai, } \\
\text { Jiangsu, } \\
\text { Jiangxi, } \\
\text { Fujian }\end{array}$ & 5 \\
\hline $\begin{array}{l}\text { Gao et al. } \\
\text { (2009) [43] }\end{array}$ & Nurses & $\begin{array}{l}32.9 \pm 8.8,20- \\
52\end{array}$ & Unknown & $1018(92.5 \%)$ & 100 & Yunnan & 5 \\
\hline $\begin{array}{l}\text { Wan et al. } \\
\text { (2009) [44] }\end{array}$ & Nurses & $\begin{array}{l}31.9 \pm 7.5^{\mathrm{a}}, 19- \\
48\end{array}$ & $0 \%$ & $499(90.7 \%)$ & 100 & Hubei & 5 \\
\hline $\begin{array}{l}\text { Li et al. } \\
\text { (2009) [45] }\end{array}$ & Nurses & $33.4 \pm 7.2^{a}$ & $0.4 \%$ & $560(94.0 \%)$ & BREF & Shaanxi & 6 \\
\hline $\begin{array}{l}\text { Zhou et al. } \\
\text { (2009) [46] }\end{array}$ & $\begin{array}{l}\text { Employees in finance, trading, technology, } \\
\text { media, etc }\end{array}$ & $\begin{array}{l}29.7 \pm 7.6,19- \\
59\end{array}$ & $35.9 \%$ & $1001(95.3 \%)$ & BREF & Shanghai & 5 \\
\hline $\begin{array}{l}\text { Zhang et al. } \\
\text { (2009) [47] }\end{array}$ & Nurses & $\begin{array}{l}31.8 \pm 8.1,18- \\
55\end{array}$ & $2.1 \%$ & $610(87.1 \%)$ & 100 & Xinjiang & 7 \\
\hline $\begin{array}{l}\text { Huang et al. } \\
\text { (2009) [48] }\end{array}$ & Construction workers & Unknown & Unknown & $\begin{array}{l}1035 \\
\text { (Unknown) }\end{array}$ & BREF & Anhui & 4 \\
\hline $\begin{array}{l}\text { Huang et al. } \\
\text { (2009) [49] }\end{array}$ & Train drivers & $\begin{array}{l}31.1 \pm 6.9,19- \\
52\end{array}$ & $100 \%$ & $230(100 \%)$ & BREF & Guangdong & 5 \\
\hline $\begin{array}{l}\text { Ding et al. } \\
\text { (2009) [50] }\end{array}$ & Construction workers & $\begin{array}{l}32.5 \pm 10.0,18- \\
50\end{array}$ & $89.1 \%$ & $101(94.4 \%)$ & BREF & Shandong & 5 \\
\hline $\begin{array}{l}\text { Song et al. } \\
\text { (2009) [51] }\end{array}$ & Journalists & Unknown & $0 \%$ & $\begin{array}{l}117 \\
\text { (Unknown) }\end{array}$ & BREF & Unknown & 3 \\
\hline $\begin{array}{l}\text { Gu et al. } \\
\text { (2009) [52] }\end{array}$ & Electronic enterprise workers & $\begin{array}{l}\text { mainly } 20-30 \\
(64.9 \%)\end{array}$ & $31.6 \%$ & $868(86.8 \%)$ & 100 & Jiangsu & 5 \\
\hline $\begin{array}{l}\text { Song et al. } \\
\text { (2009) [53] }\end{array}$ & Slaughterhouse workers & Unknown & Unknown & 970 (64.3\%) & BREF & Hebei & 4 \\
\hline $\begin{array}{l}\text { Liu et al. } \\
\text { (2009) [54] }\end{array}$ & Medical staff & $38.7 \pm 9.9^{a}$ & $26.7 \%$ & 664 (94.9\%) & BREF & Liaoning & 5 \\
\hline $\begin{array}{l}\text { Wang et al. } \\
\text { (2009) [55] }\end{array}$ & $\begin{array}{l}\text { Education, scientific research, } \\
\text { administrative management, medical } \\
\text { technology and other workers }\end{array}$ & $\begin{array}{l}48.0 \pm 5.5,40- \\
60\end{array}$ & $52.2 \%$ & $1315(84.3 \%)$ & BREF & Guizhou & 6 \\
\hline $\begin{array}{l}\text { Xing et al. } \\
\text { (2010) [56] }\end{array}$ & Nurses & $31.6 \pm 6.9$ & $5.1 \%$ & 99 (82.5\%) & BREF & Shandong & 4 \\
\hline $\begin{array}{l}\text { Bai et al. } \\
\text { (2010) [57] }\end{array}$ & Civil servants & $\begin{array}{l}36.7 \pm 8.4^{\mathrm{a}}, 20- \\
60\end{array}$ & $51.3 \%$ & $809(95.2 \%)$ & BREF & Xinjiang & 5 \\
\hline $\begin{array}{l}\text { Wang et al. } \\
\text { (2010) [58] }\end{array}$ & Medical staff & $\begin{array}{l}31.0 \pm 9.1,19- \\
70\end{array}$ & $11.4 \%$ & $\begin{array}{l}404 \\
\text { (Unknown) }\end{array}$ & BREF & Beijing & 4 \\
\hline $\begin{array}{l}\text { Fu et al. } \\
\text { (2010) [59] }\end{array}$ & Scientific research personnel & $40.0,27-56$ & $72.7 \%$ & $\begin{array}{l}260 \\
\text { (Unknown) }\end{array}$ & BREF & Guangdong & 3 \\
\hline $\begin{array}{l}\text { Liu et al. } \\
\text { (2010) [60] }\end{array}$ & Emergency nurses & $\begin{array}{l}28.9 \pm 5.8,20- \\
58\end{array}$ & $6.1 \%$ & 196 (93.3\%) & BREF & Shandong & 5 \\
\hline $\begin{array}{l}\text { Zhang et al. } \\
\text { (2010) [61] }\end{array}$ & Steel workers & $\begin{array}{l}38.1 \pm 6.6,19- \\
51\end{array}$ & $92.7 \%$ & 383 (95.8\%) & BREF & Shanxi & 5 \\
\hline $\begin{array}{l}\text { Liu et al. } \\
\text { (2010) [62] }\end{array}$ & Nurses & $\begin{array}{l}27.5 \pm 6.2,18- \\
50\end{array}$ & $3.6 \%$ & $1213(93.3 \%)$ & 100 & Guangxi & 5 \\
\hline $\begin{array}{l}\text { Jiang et al. } \\
\text { (2010) [63] }\end{array}$ & $\begin{array}{l}\text { Construction, service, processing and } \\
\text { manufacturing workers }\end{array}$ & $\begin{array}{l}24.6 \pm 4.7^{\mathrm{a}}, 16- \\
35\end{array}$ & $28.3 \%$ & $265(75.7 \%)$ & BREF & Fujian & 5 \\
\hline
\end{tabular}


Table 2 Descriptive characteristics and quality assessment of the included publications (Continued)

\begin{tabular}{|c|c|c|c|c|c|c|c|}
\hline Author(year) & Occupation & $\begin{array}{l}\text { Age (mean } \pm \\
\text { standard } \\
\text { deviation, } \\
\text { range) }\end{array}$ & $\begin{array}{l}\text { Gender } \\
\text { (\%male) }\end{array}$ & $\begin{array}{l}\text { Sample size } \\
\text { (effective } \\
\text { response } \\
\text { rate) }\end{array}$ & Questionnaire & $\begin{array}{l}\text { Region of } \\
\text { work } \\
\text { (province) }\end{array}$ & $\begin{array}{l}\text { Quality } \\
\text { assessment } \\
\text { score }\end{array}$ \\
\hline $\begin{array}{l}\text { Tang et al. } \\
\text { (2010) [64] }\end{array}$ & Elementary and middle school teachers & $22-59$ & $44.4 \%$ & $169(92.9 \%)$ & 100 & Zhejiang & 4 \\
\hline $\begin{array}{l}\text { Yao et al. } \\
\text { (2010) [65] }\end{array}$ & Medical college teachers & $36.6,24-59$ & $33.6 \%$ & $345(95.8 \%)$ & BREF & Shanxi & 5 \\
\hline $\begin{array}{l}\text { Jin et al. } \\
\text { (2011) [66] }\end{array}$ & Nurses & $\begin{array}{l}31.6 \pm 9.1^{a}, 19- \\
53\end{array}$ & $0 \%$ & $\begin{array}{l}200 \\
\text { (Unknown) }\end{array}$ & 100 & Guangdong & 3 \\
\hline $\begin{array}{l}\text { Xu et al. } \\
\text { (2011) [67] }\end{array}$ & Nurses & $35.0 \pm 8.0$ & Unknown & $561(93.5 \%)$ & BREF & Beijing & 5 \\
\hline $\begin{array}{l}\text { Lou et al. } \\
\text { (2011) [68] }\end{array}$ & Medical staff & $34.9 \pm 9.1^{\mathrm{a}}$ & $22.3 \%$ & $\begin{array}{l}452 \\
\text { (Unknown) }\end{array}$ & BREF & Shenzhen & 5 \\
\hline $\begin{array}{l}\text { Wang et al. } \\
\text { (2011) [69] }\end{array}$ & Nurses & $28.4,19-45$ & $0.3 \%$ & $385(96.7 \%)$ & BREF & Tianjin & 5 \\
\hline $\begin{array}{l}\text { Long et al. } \\
\text { (2011) [70] }\end{array}$ & Doctors & $23-60$ & $57.0 \%$ & $235(78.3 \%)$ & BREF & Guangdong & 4 \\
\hline $\begin{array}{l}\text { Wei et al. } \\
\text { (2011) [71] }\end{array}$ & Military personnel & $\begin{array}{l}21.2 \pm 2.8,18- \\
34\end{array}$ & $100 \%$ & $559(98.4 \%)$ & BREF & Unknown & 5 \\
\hline $\begin{array}{l}\text { Ye et al. } \\
\text { (2011) [72] }\end{array}$ & Military personnel & $\begin{array}{l}21.5 \pm 2.9,17- \\
33\end{array}$ & $100 \%$ & $554(90.8 \%)$ & BREF & Yunnan & 6 \\
\hline $\begin{array}{l}\text { Wan et al. } \\
\text { (2011) [73] }\end{array}$ & Policemen & Unknown & $62.9 \%$ & 70 (Unknown) & BREF & Yunnan & 2 \\
\hline $\begin{array}{l}\text { Xiong et al. } \\
\text { (2011) [74] }\end{array}$ & Medical staff & $33.4 \pm 8.0$ & $35.0 \%$ & $\begin{array}{l}331 \\
\text { (Unknown) }\end{array}$ & BREF & Hubei & 5 \\
\hline $\begin{array}{l}\text { Wang et al. } \\
\text { (2011) [75] }\end{array}$ & Medical staff & $37.0,21-60$ & $26.0 \%$ & $672(97.4 \%)$ & $\begin{array}{l}\text { WHOQOL- } \\
\text { BREF }\end{array}$ & Beijing & 6 \\
\hline $\begin{array}{l}\text { Zhang et al. } \\
\text { (2011) [76] }\end{array}$ & Medical college teachers & $37.0,21-60$ & $30.1 \%$ & 249 (88.9\%) & BREF & Anhui & 5 \\
\hline $\begin{array}{l}\text { Ma et al. } \\
\text { (2012) [77] }\end{array}$ & Military personnel & $37.6 \pm 13.1^{\mathrm{a}}$ & $100 \%$ & $181(90.5 \%)$ & BREF & Unknown & 4 \\
\hline $\begin{array}{l}\text { Ma et al. } \\
\text { (2012) [78] }\end{array}$ & Peasant workers & $26.8 \pm 4.8$ & $63.1 \%$ & $\begin{array}{l}756 \\
\text { (Unknown) }\end{array}$ & 100 & Hebei & 3 \\
\hline $\begin{array}{l}\text { Ban et al. } \\
\text { (2012) [79] }\end{array}$ & Special education teachers & Unknown & $35.9 \%$ & $131(87.3 \%)$ & BREF & Guizhou & 4 \\
\hline $\begin{array}{l}\text { Wang et al. } \\
\text { (2012) [80] }\end{array}$ & Nurses & Unknown & Unknown & $290(96.7 \%)$ & 100 & Shenzhen & 3 \\
\hline $\begin{array}{l}\text { Hu et al. } \\
\text { (2012) [81] }\end{array}$ & Enameled wire workers & $\begin{array}{l}32.5 \pm 7.2,19- \\
55\end{array}$ & $74.3 \%$ & $\begin{array}{l}319 \\
\text { (Unknown) }\end{array}$ & BREF & Anhui & 5 \\
\hline $\begin{array}{l}\text { Xu et al. } \\
\text { (2012) [82] }\end{array}$ & Nurses & $31.0,18-54$ & Unknown & $287(88.6 \%)$ & BREF & Guangdong & 4 \\
\hline $\begin{array}{l}\text { Zhang et al. } \\
\text { (2012) [83] }\end{array}$ & Medical staff & $>40$ & $21.5 \%$ & $536(97.1 \%)$ & BREF & Beijing & 6 \\
\hline $\begin{array}{l}\text { Liu et al. } \\
\text { (2012) [84] }\end{array}$ & Electronic enterprise workers & $34.9 \pm 10.8^{\mathrm{a}}$ & $10.0 \%$ & $641(98.6 \%)$ & BREF & Guangdong & 4 \\
\hline $\begin{array}{l}\text { Zhang et al. } \\
\text { (2013) [85] }\end{array}$ & Service workers & $24.3 \pm 6.2^{a}$ & $0 \%$ & $\begin{array}{l}358 \\
\text { (Unknown) }\end{array}$ & BREF & Hebei & 5 \\
\hline $\begin{array}{l}\text { Xu et al. } \\
\text { (2013) [86] }\end{array}$ & Nurses & $34.2 \pm 10.9^{a}$ & $2.0 \%$ & $256(88.6 \%)$ & BREF & Beijing & 4 \\
\hline $\begin{array}{l}\text { Wang et al. } \\
\text { (2013) [87] }\end{array}$ & Employees in public places & $\begin{array}{l}30.1 \pm 8.0,19- \\
57\end{array}$ & $27.5 \%$ & $\begin{array}{l}200 \\
\text { (Unknown) }\end{array}$ & BREF & Anhui & 4 \\
\hline $\begin{array}{l}\text { Hu et al. } \\
\text { (2013) [88] }\end{array}$ & Civil servants & $33.6 \pm 10.5$ & $55.4 \%$ & $514(93.5 \%)$ & BREF & Chongqing & 5 \\
\hline Tan et al. & Medical staff & $39.8 \pm 11.1^{\mathrm{a}}$ & Unknown & 273 & BREF & Guangdong & 2 \\
\hline
\end{tabular}


Table 2 Descriptive characteristics and quality assessment of the included publications (Continued)

\begin{tabular}{|c|c|c|c|c|c|c|c|}
\hline Author(year) & Occupation & $\begin{array}{l}\text { Age (mean } \pm \\
\text { standard } \\
\text { deviation, } \\
\text { range) }\end{array}$ & $\begin{array}{l}\text { Gender } \\
\text { (\%male) }\end{array}$ & $\begin{array}{l}\text { Sample size } \\
\text { (effective } \\
\text { response } \\
\text { rate) }\end{array}$ & Questionnaire & $\begin{array}{l}\text { Region of } \\
\text { work } \\
\text { (province) }\end{array}$ & $\begin{array}{l}\text { Quality } \\
\text { assessment } \\
\text { score }\end{array}$ \\
\hline (2013) [89] & & & & (Unknown) & & & \\
\hline $\begin{array}{l}\text { Shan et al. } \\
\text { (2013) [90] }\end{array}$ & Medical staff & $37.0 \pm 8.6$ & $54.9 \%$ & $82(82.0 \%)$ & BREF & Zhejiang & 4 \\
\hline $\begin{array}{l}\text { Wu et al. } \\
\text { (2013) [91] }\end{array}$ & Doctors & $\begin{array}{l}34.9 \pm 5.9,21- \\
48\end{array}$ & $38.1 \%$ & $291(89.8 \%)$ & BREF & Fujian & 4 \\
\hline $\begin{array}{l}\text { Xing et al. } \\
\text { (2013) [92] }\end{array}$ & $\begin{array}{l}\text { Manufacturing, food and domestic service, } \\
\text { retail sector, construction industry, } \\
\text { transportation and other workers }\end{array}$ & $\begin{array}{l}39.9 \pm 12.2^{\mathrm{a}} \\
20-65\end{array}$ & $48.4 \%$ & $1869(93.5 \%)$ & BREF & Zhejiang & 6 \\
\hline $\begin{array}{l}\text { Yu et al. } \\
\text { (2013) [93] }\end{array}$ & Nurses & $24.4 \pm 3.5$ & $10.5 \%$ & $468(78.0 \%)$ & BREF & Hunan & 6 \\
\hline $\begin{array}{l}\text { Fu et al. } \\
\text { (2013) [94] }\end{array}$ & Nurses & $\begin{array}{l}27.5 \pm 5.0,19- \\
50\end{array}$ & $0 \%$ & $310(91.2 \%)$ & 100 & Henan & 4 \\
\hline $\begin{array}{l}\text { Zhang et al. } \\
\text { (2013) [95] }\end{array}$ & Nurses & Unknown & $47.1 \%$ & $374(93.5 \%)$ & BREF & Shandong & 6 \\
\hline $\begin{array}{l}\text { Wu et al. } \\
\text { (2013) [96] }\end{array}$ & Foundry enterprise workers & $\begin{array}{l}26.4 \pm 2.8,22- \\
39\end{array}$ & $82.4 \%$ & $901(91.5 \%)$ & BREF & Anhui & 6 \\
\hline $\begin{array}{l}\text { Geng et al. } \\
\text { (2013) [97] }\end{array}$ & Nurses & $43.8 \pm 9.1^{\mathrm{a}}$ & $0 \%$ & $793(88.1 \%)$ & BREF & $\begin{array}{l}\text { Beijing and } \\
\text { Tianjin }\end{array}$ & 5 \\
\hline $\begin{array}{l}\text { Lin et al. } \\
\text { (2014) [98] }\end{array}$ & Medical staff & $\begin{array}{l}31.2 \pm 8.0,18- \\
57\end{array}$ & $0 \%$ & $315(95.5 \%)$ & BREF & Fujian & 6 \\
\hline $\begin{array}{l}\text { He et al. } \\
\text { (2014) [99] }\end{array}$ & $\begin{array}{l}\text { Peasant workers engaged in non- } \\
\text { agricultural production work }\end{array}$ & $39.2 \pm 8.8^{a}$ & $70.6 \%$ & $436(86.7 \%)$ & BREF & Unknown & 4 \\
\hline $\begin{array}{l}\text { Li et al. } \\
\text { (2014) [100] }\end{array}$ & Nurses & $18-30$ & $0 \%$ & $450(88.2 \%)$ & BREF & Henan & 6 \\
\hline $\begin{array}{l}\text { Guo et al. } \\
\text { (2014) [101] }\end{array}$ & $\begin{array}{l}\text { Network, communications, } \\
\text { pharmaceutical, banking and other } \\
\text { industries staff; mining workers; } \\
\text { construction workers }\end{array}$ & $\begin{array}{l}28.6 \pm 4.9,20- \\
46\end{array}$ & Unknown & $\begin{array}{l}1165 \\
\text { (Unknown) }\end{array}$ & BREF & Beijing & 3 \\
\hline $\begin{array}{l}\text { Li et al. } \\
\text { (2014) [102] }\end{array}$ & Nurses & $34.3 \pm 9.3$ & $0 \%$ & $356(96.2 \%)$ & BREF & Heilongjiang & 6 \\
\hline $\begin{array}{l}\text { Lao et al. } \\
\text { (2014) [103] }\end{array}$ & Doctors & $\begin{array}{l}29.5 \pm 4.0,19- \\
50\end{array}$ & $77.4 \%$ & $1064(62.6 \%)$ & BREF & Hunan & 6 \\
\hline $\begin{array}{l}\text { Wang et al. } \\
\text { (2014) [104] }\end{array}$ & Military personnel & $34.5 \pm 6.8$ & $100 \%$ & $\begin{array}{l}445 \\
\text { (Unknown) }\end{array}$ & BREF & Unknown & 4 \\
\hline $\begin{array}{l}\text { Zhang et al. } \\
\text { (2014) [105] }\end{array}$ & Community nurses & $20.7 \pm 3.0$ & $8.2 \%$ & $232(96.3 \%)$ & BREF & Jiangsu & 5 \\
\hline $\begin{array}{l}\text { Yang et al. } \\
\text { (2014) [106] }\end{array}$ & Kindergarten teachers & $\begin{array}{l}33.2 \pm 5.3,18- \\
60\end{array}$ & $14.6 \%$ & $403(91.6 \%)$ & BREF & Guizhou & 6 \\
\hline $\begin{array}{l}\text { Han et al. } \\
\text { (2014) [107] }\end{array}$ & Nurses & $\begin{array}{l}28.0 \pm 8.0,16- \\
50\end{array}$ & $0 \%$ & $102(92.7 \%)$ & BREF & Shanghai & 4 \\
\hline $\begin{array}{l}\text { Wu et al. } \\
\text { (2014) [108] }\end{array}$ & Nurses & $\begin{array}{l}28.4 \pm 5.5,22- \\
48\end{array}$ & $0 \%$ & 215 (97.7\%) & BREF & Henan & 4 \\
\hline $\begin{array}{l}\text { Zhang et al. } \\
\text { (2015) [109] }\end{array}$ & Nurses & $\begin{array}{l}28.9 \pm 7.8,20- \\
48\end{array}$ & $36.5 \%$ & $181(97.8 \%)$ & BREF & Shandong & 5 \\
\hline $\begin{array}{l}\text { Yang et al. } \\
\text { (2015) [110] }\end{array}$ & $\begin{array}{l}\text { HIV / AIDS prevention and control } \\
\text { personnel }\end{array}$ & $28.8,23-48$ & $31.6 \%$ & $250(100 \%)$ & BREF & Guangxi & 5 \\
\hline $\begin{array}{l}\text { Guan et al. } \\
\text { (2015) [111] }\end{array}$ & $\begin{array}{l}\text { HIV / AIDS prevention and control } \\
\text { personnel }\end{array}$ & $\begin{array}{l}32.5 \pm 8.4,19- \\
60\end{array}$ & $46.0 \%$ & $250(100 \%)$ & BREF & Heilongjiang & 5 \\
\hline $\begin{array}{l}\text { Li et al. } \\
\text { (2015) [112] }\end{array}$ & Medical staff & $\begin{array}{l}39.7 \pm 8.6,21- \\
63\end{array}$ & $2.6 \%$ & 76 (Unknown) & BREF & Henan & 4 \\
\hline $\begin{array}{l}\text { Jiang et al. } \\
\text { (2015) [113] }\end{array}$ & Railway construction workers & $\begin{array}{l}29.1 \pm 10.9,22- \\
45\end{array}$ & $98.3 \%$ & $950(94.0 \%)$ & BREF & Shanxi & 6 \\
\hline
\end{tabular}


Table 2 Descriptive characteristics and quality assessment of the included publications (Continued)

\begin{tabular}{|c|c|c|c|c|c|c|c|}
\hline Author(year) & Occupation & $\begin{array}{l}\text { Age (mean } \pm \\
\text { standard } \\
\text { deviation, } \\
\text { range) }\end{array}$ & $\begin{array}{l}\text { Gender } \\
\text { (\%male) }\end{array}$ & $\begin{array}{l}\text { Sample size } \\
\text { (effective } \\
\text { response } \\
\text { rate) }\end{array}$ & Questionnaire & $\begin{array}{l}\text { Region of } \\
\text { work } \\
\text { (province) }\end{array}$ & $\begin{array}{l}\text { Quality } \\
\text { assessment } \\
\text { score }\end{array}$ \\
\hline $\begin{array}{l}\text { Miao et al. } \\
\text { (2015) [114] }\end{array}$ & Nurses & $\begin{array}{l}29.4 \pm 11.6,24- \\
44\end{array}$ & Unknown & $268(95.7 \%)$ & BREF & Heilongjiang & 4 \\
\hline $\begin{array}{l}\text { Tang et al. } \\
(2015) \text { [115] }\end{array}$ & Doctors & $\begin{array}{l}39.9 \pm 11.3^{\mathrm{a}} \\
15-65\end{array}$ & $51.7 \%$ & $576(91.4 \%)$ & BREF & Guangdong & 6 \\
\hline $\begin{array}{l}\text { Kang et al. } \\
\text { (2015) [116] }\end{array}$ & Medical rescuers & $31.4 \pm 6.9^{a}$ & $33.7 \%$ & $303(89.6 \%)$ & BREF & Gansu & 7 \\
\hline $\begin{array}{l}\text { Yan et al. } \\
\text { (2015) [117] }\end{array}$ & Doctors & $40.2 \pm 8.5$ & $90.0 \%$ & $60(96.8 \%)$ & BREF & Guangdong & 4 \\
\hline $\begin{array}{l}\text { Pan et al. } \\
\text { (2015) [118] }\end{array}$ & Nurses & $32.6 \pm 7.3$ & $11.8 \%$ & $152(95.0 \%)$ & BREF & Guangdong & 4 \\
\hline $\begin{array}{l}\text { Chen et al. } \\
\text { (2016) [119] }\end{array}$ & Sanitation workers & $32.8 \pm 12.9^{\mathrm{a}}$ & $43.8 \%$ & $121(63.0 \%)$ & BREF & Ningxia & 4 \\
\hline $\begin{array}{l}\text { Dai et al. } \\
\text { (2016) [120] }\end{array}$ & Civil servants & $\begin{array}{l}32.7 \pm 8.6,19- \\
54\end{array}$ & $57.5 \%$ & $708(79.8 \%)$ & BREF & Jiangsu & 5 \\
\hline $\begin{array}{l}\text { Hu et al. } \\
\text { (2016) [121] }\end{array}$ & Workers in a chemical enterprise & $\begin{array}{l}51.1 \pm 9.7^{\mathrm{a}}, 30- \\
70\end{array}$ & $71.4 \%$ & $538(90.7 \%)$ & BREF & Anhui & 6 \\
\hline $\begin{array}{l}\text { Yang et al. } \\
\text { (2016) [122] }\end{array}$ & $\begin{array}{l}\text { Workers in nonferrous metal ore } \\
\text { concentrator, smelting enterprise, lead } \\
\text { acid battery enterprise }\end{array}$ & $\begin{array}{l}35.8 \pm 9.5,21- \\
59\end{array}$ & $0 \%$ & $652(97.3 \%)$ & BREF & Guangdong & 5 \\
\hline $\begin{array}{l}\text { Zhao et al. } \\
\text { (2016) [123] }\end{array}$ & Military personnel & $\begin{array}{l}40.9 \pm 10.1^{a} \\
18-59\end{array}$ & $87.5 \%$ & $616(94.8 \%)$ & BREF & Unknown & 5 \\
\hline $\begin{array}{l}\text { Tang et al. } \\
\text { (2017) [124] }\end{array}$ & Nurses & $\begin{array}{l}39.9 \pm 9.1^{\mathrm{a}}, 22- \\
54\end{array}$ & Unknown & 40 (Unknown) & 100 & Liaoning & 2 \\
\hline $\begin{array}{l}\text { Zhang et al. } \\
\text { (2017) [125] }\end{array}$ & Medical staff & $\begin{array}{l}22.6 \pm 4.9,17- \\
47\end{array}$ & $37.7 \%$ & 239 (95.2\%) & BREF & Tibet & 5 \\
\hline $\begin{array}{l}\text { Lai et al. } \\
(2017)[126]\end{array}$ & Nurses & $32.1 \pm 9.0^{\mathrm{a}}$ & $0 \%$ & $\begin{array}{l}100 \\
\text { (Unknown) }\end{array}$ & BREF & Shenzhen & 3 \\
\hline $\begin{array}{l}\text { Zhao et al. } \\
\text { (2017) [127] }\end{array}$ & Medical staff & $\begin{array}{l}35.5 \pm 5.1,20- \\
50\end{array}$ & Unknown & 406 (81.2\%) & BREF & Shaanixi & 5 \\
\hline $\begin{array}{l}\text { Xiao et al. } \\
\text { (2017) [128] }\end{array}$ & Seafarers & Unknown & $100 \%$ & 917 (98.7\%) & BREF & Jiangsu & 6 \\
\hline $\begin{array}{l}\text { Su et al. } \\
(2017)[129]\end{array}$ & Armed polices & $33.5 \pm 9.6$ & $100 \%$ & 1327 (95.8\%) & BREF & Shanxi & 6 \\
\hline $\begin{array}{l}\text { Liu et al. } \\
\text { (2017) [130] }\end{array}$ & Doctors & $\begin{array}{l}21.0 \pm 1.4,17- \\
34\end{array}$ & $68.1 \%$ & $276(92.3 \%)$ & BREF & Hubei & 4 \\
\hline $\begin{array}{l}\text { Zhang et al. } \\
\text { (2017) [131] }\end{array}$ & Coal workers & $45.9 \pm 11.1^{\mathrm{a}}$ & $63.7 \%$ & 881 (97.9\%) & BREF & Shanxi & 7 \\
\hline $\begin{array}{l}\text { Yi et al. } \\
\text { (2018) [132] }\end{array}$ & Coal miners & $\begin{array}{l}37.7 \pm 8.5,18- \\
65\end{array}$ & Unknown & $263(87.7 \%)$ & BREF & Henan & 4 \\
\hline $\begin{array}{l}\text { Zeng et al. } \\
\text { (2018) [133] }\end{array}$ & Military personnel & $38.7 \pm 7.9$ & $100 \%$ & $154(96.3 \%)$ & BREF & Unknown & 4 \\
\hline $\begin{array}{l}\text { Yang et al. } \\
\text { (2018) [134] }\end{array}$ & Service workers & $24.9 \pm 3.8$ & $26.6 \%$ & $\begin{array}{l}139 \\
\text { (Unknown) }\end{array}$ & BREF & Yunnan & 3 \\
\hline $\begin{array}{l}\text { Lu et al. } \\
\text { (2018) [135] }\end{array}$ & $\begin{array}{l}\text { Migrant workers in Construction industry, } \\
\text { catering industry, etc }\end{array}$ & $\begin{array}{l}31.1 \pm 9.7^{\mathrm{a}}, 16- \\
56\end{array}$ & $55.4 \%$ & 267 (95.7\%) & BREF & Tianjin & 4 \\
\hline $\begin{array}{l}\text { Zhao et al. } \\
\text { (2018) [136] }\end{array}$ & Nurses & $\begin{array}{l}25.9 \pm 4.7^{\mathrm{a}}, 18- \\
36\end{array}$ & Unknown & $282(95.6 \%)$ & BREF & Hebei & 4 \\
\hline $\begin{array}{l}\text { Xue et al. } \\
\text { (2018) [137] }\end{array}$ & Nurses & $36.8 \pm 9.7^{a}$ & $0 \%$ & 400 (87.0\%) & BREF & Jiangsu & 6 \\
\hline $\begin{array}{l}\text { Song et al. } \\
\text { (2018) [138] }\end{array}$ & Medical staff & $32.8 \pm 12.9^{a}$ & $23.2 \%$ & 2274 (91.0\%) & BREF & Beijing & 5 \\
\hline
\end{tabular}


Table 2 Descriptive characteristics and quality assessment of the included publications (Continued)

\begin{tabular}{|c|c|c|c|c|c|c|c|}
\hline Author(year) & Occupation & $\begin{array}{l}\text { Age (mean } \pm \\
\text { standard } \\
\text { deviation, } \\
\text { range) }\end{array}$ & $\begin{array}{l}\text { Gender } \\
\text { (\%male) }\end{array}$ & $\begin{array}{l}\text { Sample size } \\
\text { (effective } \\
\text { response } \\
\text { rate) }\end{array}$ & Questionnaire & $\begin{array}{l}\text { Region of } \\
\text { work } \\
\text { (province) }\end{array}$ & $\begin{array}{l}\text { Quality } \\
\text { assessment } \\
\text { score }\end{array}$ \\
\hline $\begin{array}{l}\text { Yang et al. } \\
\text { (2018) [139] }\end{array}$ & University teachers & $36.0,20-70$ & $47.0 \%$ & $25,066(78.3 \%)$ & BREF & Unknown & 7 \\
\hline $\begin{array}{l}\text { Yu et al. } \\
\text { (2019) [140] }\end{array}$ & Nurses and other medical staffs & $\begin{array}{l}37.2 \pm 7.8^{\mathrm{a}}, 24- \\
65\end{array}$ & $29.6 \%$ & $\begin{array}{l}230 \\
\text { (Unknown) }\end{array}$ & BREF & Fujian & 3 \\
\hline $\begin{array}{l}\text { He et al. } \\
\text { (2019) [141] }\end{array}$ & Nurses and other medical staffs & $\begin{array}{l}38.0 \pm 3.2,30- \\
46\end{array}$ & $18.5 \%$ & $\begin{array}{l}200 \\
\text { (Unknown) }\end{array}$ & BREF & Hebei & 3 \\
\hline $\begin{array}{l}\text { Song et al. } \\
\text { (2019) [142] }\end{array}$ & Nurses & $\begin{array}{l}31.1 \pm 3.4,22- \\
45\end{array}$ & $0 \%$ & $558(93.0 \%)$ & BREF & Liaoning & 5 \\
\hline $\begin{array}{l}\text { Ma et al. } \\
\text { (2019) [143] }\end{array}$ & Coal workers & Unknown & $84.2 \%$ & $3090(71.2 \%)$ & BREF & Shanxi & 6 \\
\hline $\begin{array}{l}\text { Asante et al. } \\
\text { (2019) [144] }\end{array}$ & Primary healthcare workers & $\begin{array}{l}51.7 \pm 12.6^{a} \\
20-65\end{array}$ & $50.9 \%$ & $873(87.3 \%)$ & BREF & Guangdong & 6 \\
\hline $\begin{array}{l}\text { Zhu et al. } \\
\text { (2019) [145] }\end{array}$ & Nurses & $32.4 \pm 6.9^{\mathrm{a}}$ & $100 \%$ & $315(95.5 \%)$ & BREF & Shandong & 6 \\
\hline $\begin{array}{l}\text { Wu et al. } \\
\text { (2020) [146] }\end{array}$ & Fishermen & $27.9 \pm 5.6^{\mathrm{a}}$ & $99.4 \%$ & $\begin{array}{l}507 \\
\text { (Unknown) }\end{array}$ & BREF & Hainan & 5 \\
\hline $\begin{array}{l}\text { Zeng et al. } \\
\text { (2020) [147] }\end{array}$ & Nurses & $\begin{array}{l}36.9 \pm 11.3,16- \\
66\end{array}$ & $80.5 \%$ & $1449(68.2 \%)$ & BREF & Unknown & 5 \\
\hline $\begin{array}{l}\text { Liu et al. } \\
\text { (2020) [148] }\end{array}$ & Nurses & $32.6 \pm 8.8$ & $9.3 \%$ & 75 (Unknown) & BREF & Tianjin & 3 \\
\hline $\begin{array}{l}\text { Luo et al. } \\
\text { (2020) [149] }\end{array}$ & White-collar workers & $\begin{array}{l}29.1 \pm 6.2,21- \\
40\end{array}$ & $28.0 \%$ & $\begin{array}{l}410 \\
\text { (Unknown) }\end{array}$ & BREF & Zhejiang & 5 \\
\hline $\begin{array}{l}\text { Wang et al. } \\
\text { (2020) [150] }\end{array}$ & Military personnel & $34.3 \pm 9.2$ & $100 \%$ & $146(97.3 \%)$ & BREF & Unknown & 4 \\
\hline $\begin{array}{l}\text { Wei et al. } \\
\text { (2020) [151] }\end{array}$ & Pediatricians and Pediatric Nurses & $24.3 \pm 4.0$ & $11.8 \%$ & $355(93.4 \%)$ & BREF & Henan & 6 \\
\hline $\begin{array}{l}\text { Chen et al. } \\
\text { (2021) [152] }\end{array}$ & Radiation workers & $32.2 \pm 8.3^{\mathrm{a}}$ & $69.9 \%$ & $449(89.8 \%)$ & BREF & Guangdong & 5 \\
\hline
\end{tabular}

${ }^{a}$ Represents that mean age and standard deviation of this publication was estimated by age frequency

to 15.0 (95\%CI: $14.6-15.5)$. The sensitivity analysis demonstrated that when removing any one publication, the pooled scores were not altered significantly, with the overall changes differing only by $0.03(0.2 \%), 0.02(0.2 \%)$, $0.03(0.2 \%), 0.05(0.4 \%), 0.14(0.9 \%)$, and $0.15(1.2 \%)$ in the physical, psychological, social relationship, environment, independence and spirituality beliefs domain respectively.

\section{Subgroup analysis}

The data in the included publications were further analyzed by gender, age, occupation and region. Publications presenting multiple subgroups were included in the subgroup meta-analysis if the scores were reported for the respective subgroups. The gender characters were categorized into three based on gender dominance: male-dominated (>80\%, $n=49)$, female-dominated (> $80 \%, n=60)$, and mixed $(n=34) .17$ publications did not report participants' gender and 22 publications reported age-specific results. The mean age of participants was divided into 3 categories: 19.8-29.9 $(n=38), 30.0-39.9$ $(n=72)$, and 40.0-66.5 $(n=8)$. Twenty publications did not report the mean age or sample size of each age group. The occupations were grouped into three: workers in mining, construction and manufacturing were classified as blue-collar workers $(n=51)$; education, logistics and company staff as office workers $(n=20)$; and doctors, nurses and medical rescue workers as health care workers $(n=70)$. In addition, 2 publications reported occupation-specific results. Besides, we divided China into 8 geographical regions: central $(n=16)$, north $(n=26)$, east $(n=33)$, south $(n=25)$, southwest $(n=13)$, northeast $(n=6)$, northwest $(n=7)$. Twelve publications did not report the study region.

The pooled mean scores and 95\% confidence intervals for the four HRQOL domains for each subgroup are presented in Table 3 . No significant differences were found among different gender, age, and occupation groups, so these factors could not be regarded as sources of heterogeneity. The differences among regions were mainly reflected in social relationships and environmental domain. The pooled score of social relationship 


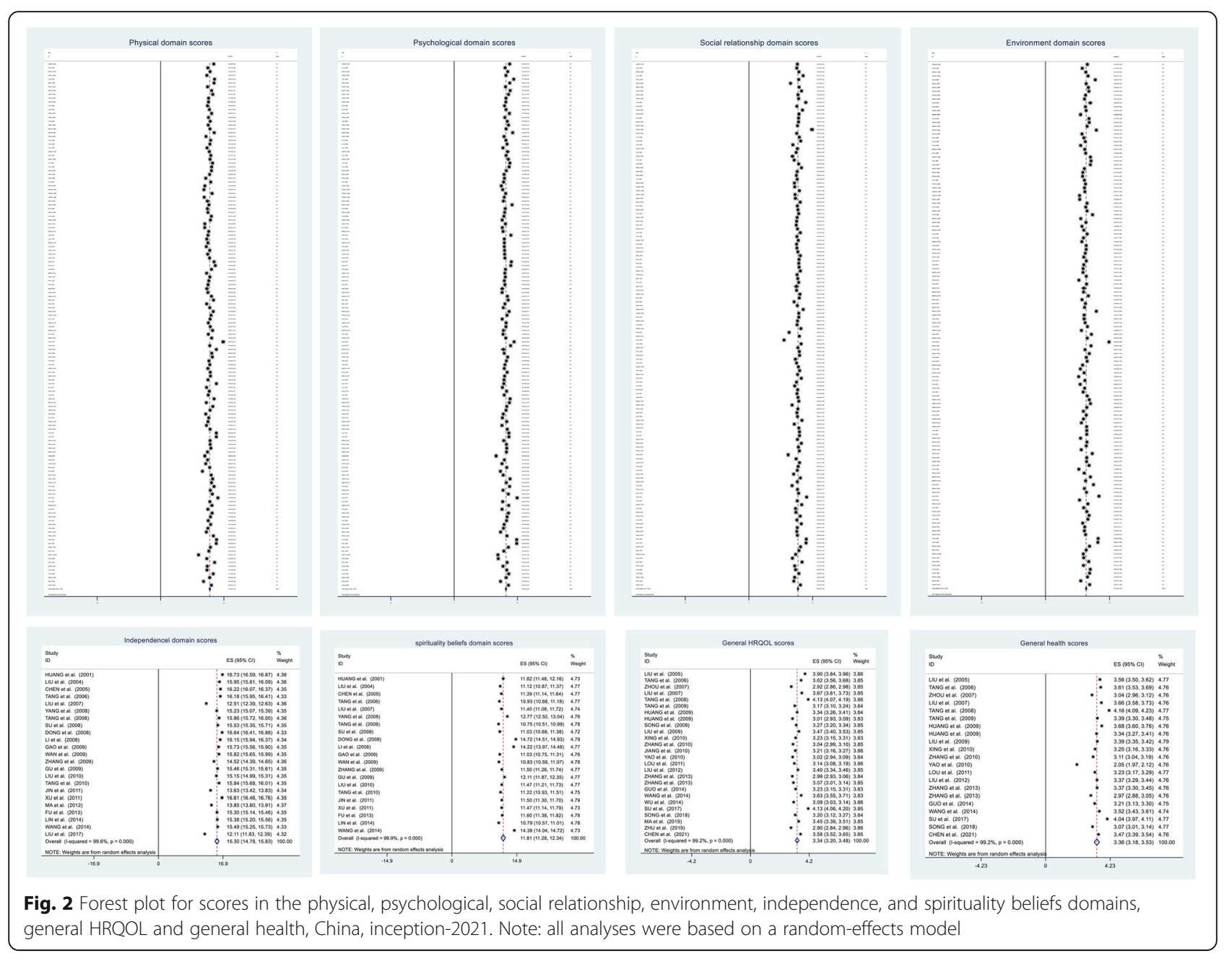

domain in northeast China was higher than that of other regions, while the pooled score of environmental domain in Central china was lower than that of other regions.

\section{Discussion}

Meta-analysis is increasingly being utilized in the health field. Meta-analysis of studies without control groups, with continuous outcome variables, only provide data for specific populations. Although investigations of HRQOL in different occupational groups have been conducted in several regions of China, there is great variation by gender, age, occupation and sample size, and these discrete results may therefore be difficult to compare between studies or use as an index to reflect changes after implantation of policy or health program. Therefore, we systematically reviewed the literature on occupational quality of life and attempted to estimate combined scores.

A total of 139 publications were included in metaanalysis, of which 136 were based on cross sectional studies, and 3 on intervention studies. Similar to most studies [153], the results of our meta-analysis on Chinese occupational groups showed that higher scores were found in the physical and social relationship domain than in the psychological domain, followed by the environment domain. Compared to the results from two large surveys conducted in China, a survey of 777 healthy participants [2] and a survey of 83,666 adults [154], our pooled scores were lower in all four HRQOL domains. This may be due to the difference between our study and the two surveys in sampling strategies. The two surveys targeted general adults which may have some systematic difference in occupation distribution. However, this difference could not be verified because these two surveys did not report data on occupation composition. The publications we included were presenting vulnerable occupational groups with impaired well-being, such as workers with heavy physical load, high work intensity and high psychological pressure.

Although subgroup analyses were conducted to explore the source of heterogeneity, no statistical differences were found among gender, age, and occupation 

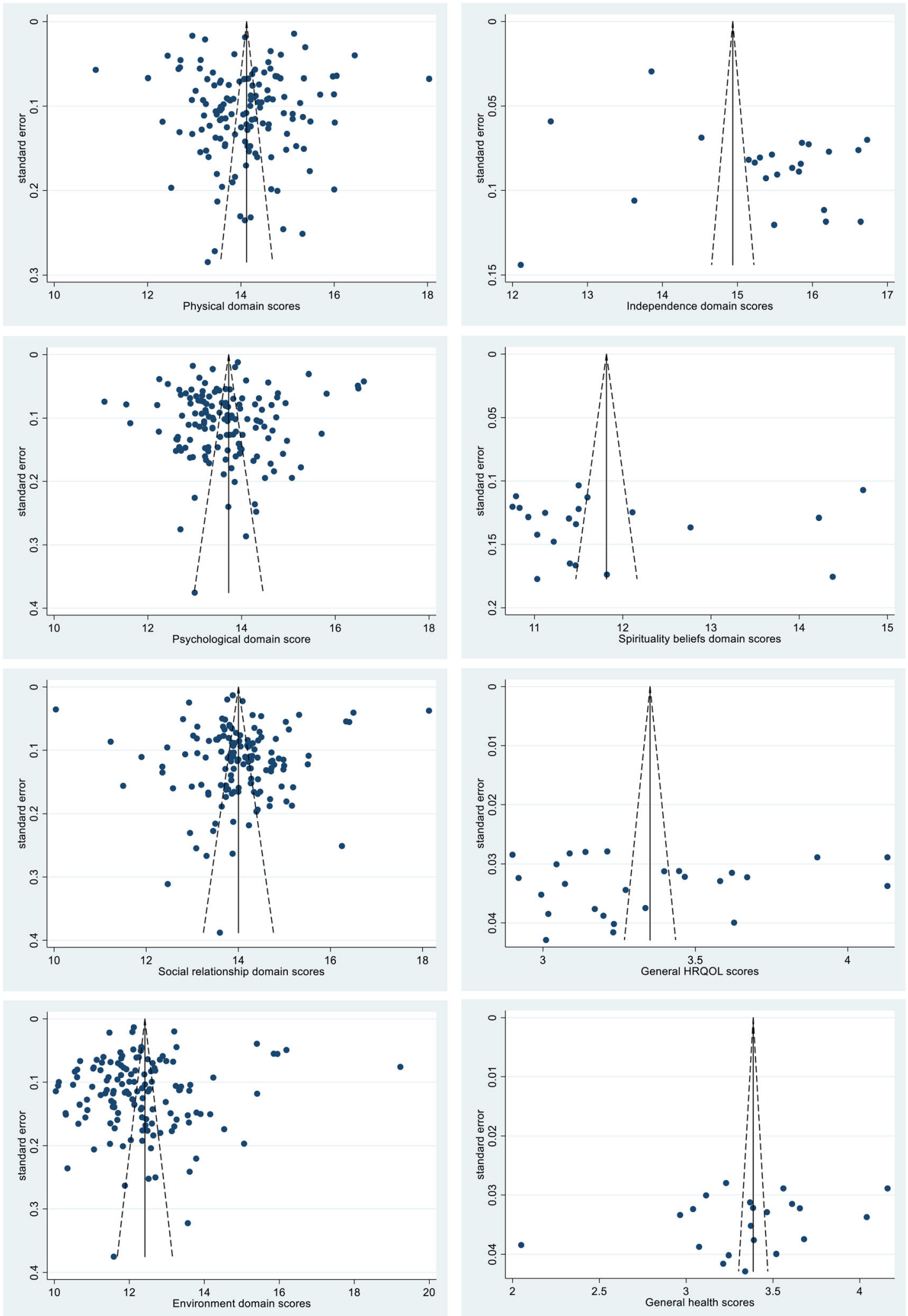

Fig. 3 Funnel plots for selected indicators of HRQOL, China, inception-2021 
Table 3 Subgroup analyses: effect size by study characteristics

\begin{tabular}{|c|c|c|c|c|}
\hline Subgroup & Physical domain & Psychological domain & Social relationship domain & Environmental domain \\
\hline \multicolumn{5}{|l|}{ Gender } \\
\hline Male-dominated & $14.0(13.7-14.3)$ & $13.6(13.3-13.8)$ & $13.8(13.5-14.0)$ & $12.4(12.0-12.8)$ \\
\hline Female-dominated & $14.2(13.8-14.5)$ & $13.6(13.3-14.0)$ & $13.8(13.4-14.3)$ & $12.2(11.7-12.7)$ \\
\hline Mixed & $14.1(13.8-14.5)$ & $13.6(13.5-13.8)$ & $13.9(13.7-14.1)$ & $12.3(12.1-12.5)$ \\
\hline \multicolumn{5}{|l|}{ Age } \\
\hline 19.8-29.9 & $14.1(13.7-14.5)$ & $13.7(13.4-14.1)$ & $14.1(13.7-14.4)$ & $12.3(11.9-12.8)$ \\
\hline $30.0-39.9$ & $14.2(13.9-14.5)$ & $13.7(13.5-13.9)$ & $13.9(13.6-14.2)$ & $12.4(12.0-12.7)$ \\
\hline $40.0-66.5$ & $13.9(13.3-14.6)$ & $13.4(12.8-13.8)$ & $14.0(13.8-14.2)$ & $12.3(12.1-12.5)$ \\
\hline \multicolumn{5}{|l|}{ Occupation } \\
\hline Manual workers & $14.3(13.9-14.5)$ & $13.8(13.5-14.1)$ & $14.2(13.7-14.6)$ & $12.3(11.9-12.7)$ \\
\hline Office workers & $14.0(13.8-14.3)$ & $13.5(13.3-13.8)$ & $13.9(13.7-14.2)$ & $12.3(12.0-12.6)$ \\
\hline Health care workers & $14.2(13.7-14.7)$ & $13.7(13.5-13.8)$ & $14.0(13.8-14.2)$ & $12.4(11.8-13.0)$ \\
\hline \multicolumn{5}{|l|}{ Region } \\
\hline Central China & $14.1(13.5-14.7)$ & $13.4(13.1-13.8)$ & $13.7(13.5-14.0)$ & $11.7(11.3-12.1)$ \\
\hline North China & $14.1(13.7-14.5)$ & $13.7(13.2-14.2)$ & $14.2(13.6-14.7)$ & $12.3(11.7-12.9)$ \\
\hline East China & $14.1(13.8-14.4)$ & $13.6(13.3-13.9)$ & $14.2(13.9-14.4)$ & $12.1(11.7-12.5)$ \\
\hline South China & $14.1(13.6-14.6)$ & $13.7(13.3-14.1)$ & $13.9(13.6-14.3)$ & 12.6 (12.2-13.0) \\
\hline Southwest China & $14.4(13.6-15.3)$ & $13.8(13.1-14.4)$ & $13.3(12.1-14.5)$ & $13.0(11.2-14.8)$ \\
\hline Northeast China & $14.1(13.7-14.4)$ & $13.6(13.2-14.0)$ & $14.7(14.2-15.2)$ & 12.9 (12.0-13.8) \\
\hline Northwest & 13.6 (13.4-13.8) & 13.6 (13.4-13.8) & $14.0(13.8-14.2)$ & $11.8(11.2-12.4)$ \\
\hline
\end{tabular}

groups. Different results across regions might be due to the differences in population distribution and resource allocation. Central China, including Hubei, Hunan, and Henan province, is a densely populated area, thus per capita resources in transportation, living conditions and medical services are relatively less. However, the influence of differences in occupation distribution cannot be excluded.

All the above three occupational groups have their own specific occupational risk factors associated with poor HRQOL. Previous studies have shown that bluecollar workers often have harsh working conditions including ergonomic, environmental and psychological hazards. For example, heavy physical load, awkward working postures, vibration, extreme temperatures, noise, harmful chemicals were correlated with musculoskeletal disorders, heat-related illness, skin and lung diseases, and can lead to poor physical health [155-158]. A higher incidence of non-fatal work injuries and fatalities has also been seen among blue-collar workers, especially construction workers. From 2014 to 2018, 3024 municipal work accidents were reported in China, resulting in an average of 717 deaths per year in China's construction industry [159]. Moreover, supervisor and coworkers support in the work environment were found to be essential predictors of the psychological health, social relationship and environmental domains of HRQOL [160].
For office workers, lack of ergonomic-featured office equipment, sitting, standing and watching computer screens for a long period, and lack of exercise, were related to arm, neck, shoulder and lower limbs pains as well as eye problems [161-163]. In addition, due to the low requirements for physical burden, office workers' on-boarding health screening may be not as strict as blue-collar workers, and were less likely to quit work because of acute injuries. For health care workers, increased number of hospital visits by an aging population, strained doctor-patient relationships, and poor sleep habits are important detrimental factors for physical and psychological health, which can lead to occupational stress, depression, burnout and physical exhaustion [144].

Sex work is illegal and not considered as an occupation in China, therefore related publications were not included in our study. Jiang et al. found that female sex workers reported lower scores than women in general in the social relationship and environment domain, which was ascribed to high population mobility and lack of occupational safety and health services. Wang et al. [164] reported lower scores for sex workers than for the general population in the physical domain, which might be related to multiple sexual partners.

As a health indicator, the assessment of quality of life makes it possible to prospectively study of diseases. Our 
study summarizes overall HRQOL levels among Chinese occupational groups and provided a potential reference for future study. Based on our study, it appears that there remains a need to strengthen the occupational safety and health management of vulnerable occupational groups and reduce exposure to known health risk factors in the future. Government departments also need to rationally allocate resources such as medical care, housing conditions and transportation according to regional factors like economic development level, industrial distribution and employment status, etc. However, close observing the trend of HRQOL over time and identifying essential contributors in the next step are imperative for relevant policy planning.

The results of our study may be biased. The study quality of the included publications was often not satisfactory because of improper sampling methods and unverified reliability and validity. Besides, about half of the included publications focused on medical staff, thus the pooled scores might be close to their results. There are also some publications that reported the results of subgroups (such as migrant workers and urban workers) rather than the entire study population, and the combination of data may induce bias. In Chinese culture, endurance was considered as a merit and people tend to underreport their discomfort. In addition, people also prefer to choose medium instead of extreme figures, which may result in similar results.

There are several limitations to the study. First, the absence of blinding (author and publication information disclosed) used in the search and selection of publications may have leaded to researcher bias. Second, although the search strategy was comprehensive, there may still have been additional studies not indexed by the selected database. Third, given the difficulties in comparing results based on different HRQOL instruments, our systematic review excluded studies that used other instruments (such as 36-Item Short-Form Health Survey, the symptom checklist-90) than WHOQOL-100 or WHOQOL-BREF. Fourth, of the included publications, some did not report the average age, gender and occupation of the participants, which may represent a group of workers with distinct results and lead to bias for subgroup analysis.

\section{Conclusion}

This is the first systematic review to synthesize the HRQOL scores for Chinese workers. The pooled scores in HRQOL were lower than those in the general population. Subgroup analysis did not suggest a strong relationship of gender, age and types of job with HRQOL scores, and region might be a source of heterogeneity. We suggest that future HRQOL studies pay more attention to these factors so that effective occupational safety and health targeted to specific groups can be developed and implemented.

\begin{abstract}
Abbreviations
HRQOL: Health-related quality of life; WHOQOL: The World Health

Organization Quality of Life; CNKI: China National Knowledge Infrastructure; WF: WanFang Data; CQVIP: China Science and Technology Journal Database; MeSH: Medical subject heading; PRISMA: Preferred reporting items for systematic reviews and meta-analyses; WHO: World Health Organization
\end{abstract}

\section{Acknowledgements}

Not applicable.

\section{Authors' contributions}

Ke-Zhi Jin and Ya Su designed the study. Ya Su and Meng-Shuang Liu reviewed the relevant articles and extracted important data. Ya Su analyzed the data and drafted the manuscript. All authors contributed to the interpretation of the findings and manuscript revision. All authors read and approved the final manuscript.

\section{Funding}

None.

\section{Declarations}

Ethics approval and consent to participate

Not applicable.

Consent for publication

Not applicable.

\section{Competing interests}

The authors declare that they have no competing interests.

\section{Author details}

${ }^{1}$ Department of Occupational Health, School of Public Health, Fudan University, 138 Yixueyuan Road Box288\#, Shanghai 200032, P.R. China. ${ }^{2}$ Key Laboratory of Public Health Safety, Ministry of Education, Shanghai, P.R. China. ${ }^{3}$ Fudan Global Health Institute, Fudan University, Shanghai, P.R. China. ${ }^{4}$ Department of Community Medicine, University of Ruhuna, Galle, Sri Lanka. ${ }^{5}$ Duke Global Health Institute, Durham, NC, USA.

Received: 15 December 2020 Accepted: 19 June 2021

Published online: 13 August 2021

References

1. WHOQOL Group. Development of the WHOQOL: rationale and current status. Int J Ment Health. 1994;23(3):24-56.

2. Fang JQ, Hao YT, Li CX. Reliability and validity for Chinese version of WHO quality of life scale. Chin Ment Health J. 1999;13(04):203-205.

3. Yuantao $\mathrm{H}$, Jiqian F. Introduction to the Chinese version of the World Health Organization Quality of life scale and its instructions. Mod Rehabil. 2000;4(8):1127-1129+1145.

4. Shockey TM, Luckhaupt SE, Groenewold MR, Lu ML. Frequent exertion and frequent standing at work, by industry and occupation group-United States, 2015. MMWR Morb Mortal Wkly Rep. 2018;67(1):1-6.

5. Jun D, Zoe M, Johnston V, O'Leary S. Physical risk factors for developing non-specific neck pain in office workers: a systematic review and metaanalysis. Int Arch Occup Environ Health. 2017:90(5):373-410.

6. Zhao Y, Richardson A, Poyser C, Butterworth P, Strazdins L, Leach LS. Shift work and mental health: a systematic review and meta-analysis. Int Arch Occup Environ Health. 2019;92(6):763-93.

7. Moreno CRC, Marqueze EC, Sargent C, Wright KP Jr, Ferguson SA, Tucker P. Working time society consensus statements: evidence-based effects of shift work on physical and mental health. Ind Health. 2019;57(2):139-57.

8. Moher D, Liberati A, Tetzlaff J, Altman DG. Reprint-preferred reporting items for systematic reviews and meta-analyses: the PRISMA statement. Phys Ther. 2009;89(9):873-80.

9. Crombie IK. The pocket guide to critical appraisal. London: BMJ Books; 1996.

10. Golriz S, Walker B. Backpacks. Several factors likely to influence design and usage: a systematic literature review. Work. 2012;42(4):519-31. 
11. Janakiraman B, Ravichandran H, Demeke S, Fasika S. Reported influences of backpack loads on postural deviation among school children: a systematic review. J Educ Health Promot. 2017;6(1):41.

12. Steele E, Bialocerkowski A, Grimmer K. The postural effects of load carriage on young people-a systematic review. BMC Musculoskelet Disord. 2003;4(1): 12.

13. World Health Organization. Programme on mental health: WHOQOL user manual. Geneva: World Health Organization; 1998.

14. Huang YL, Chen GM, Bi YY. The quality of life of nurses. J Math Med. 2001; (02):187-9.

15. Liu QW, Xiao SY, Xiong B, Huang ZP, Shi YQ, Luo YH, et al. A study on the present situation and related factors of quality of life of the medical practitioners in Chenzhou. Chin Prev Med. 2004;(02):34-7.

16. Wang Y. Study on quality of life and its influencing factors among military personnel stationed at the frontier of inner mongolia desert. master. Guangzhou: Southern Medical University; 2005

17. Chen L, Yang CT, Jiang WF, Zhou J, Hua WC. An investigation on status quo of working stress and quality of life of nurses in hospitals of Changzhou area. Chin Nurs Res. 2005:(16):1437-40

18. Li YG, Zhang WJ, Yan J, Wu YH, Xiang Q, Liu HC, et al. Analysis on the quality of life of military convalescents and its influencing factors. Chin J Convalescent Med. 2005;(01):16-8.

19. Jing CX, Wang SY, Wu CP, Lin HS, Chen QS, Liu GN. Canonical correlation analysis on oculists' quality of life. Chin J Public Health. 2005;(06):722-3.

20. Zhao JB, Jie YN, Shi MC, Ren ZW, Feng XH. Relationship between sleep quality and life quality in military personnel in high altitude area. Chin J Public Health. 2006;(06):665-6.

21. Geng RY, Jie YN, Yang Y, Zhao JB, Fang F, Hao HJ, et al. A study of the quality of sleep and the quality of life in the Chinese people's armed police forces of Guangdong province. Chin J Clin Psychol. 2006;(03):306-307+305.

22. Tang MX, Guo Q, Yan XY, Guo PF, Cai XJ. Results and analysis: a pilot study on quality of life for soldiery army. Hosp Adm J Chin People's Liberation Army. 2006;(04):325-8.

23. Tang CX, Lai FH, Liu XQ, Li SJ, Luo JY. The living quality investigation of the temporary engaged workers of state-owned hospital in certain district of Shenzhen. Mod Hosp. 2006;10:105-8.

24. Yang L. Research on psychological well-being of vocational teachers and its influencing factors. master. Shijiazhuang: Hebei Normal University; 2006.

25. Liu YN, Lin P, Man J. Investigation on quality of life of nurses in tertiary hospitals. Chin J Pract Nurs. 2007;(09):65-7.

26. Liu KJ, Kou AJ, Wu JH, Jiang XH, Liu FQ, Zhang $Y Z$, et al. Investigation of subjective fatigue and life quality of roadmen. Ind Health Occup Dis. 2007; (05):266-70

27. Chen R. Assessment on quality of life to nurses at different grade hospitals master. Kunming: Kunming Medical University; 2007.

28. Zhou SP, Liu QW, Zhou Y, Gao JX, Li J, Liang X, et al. The quality of life of primary middle school teachers and its affecting factors in Chenzhou. J Xiangnan Univ (Med Sci). 2007;(04):1-3+6.

29. Liu XQ. Study on quality of life and its influencing factors among armed police forces at qinghai high altitude: master. Guangzhou: Southern Medical University; 2007.

30. Yang $\mathrm{XH}$, Yang $\mathrm{HN}$, Xiong $\mathrm{HQ}$, Tang YP. Analysis of the health related quality of life of staff in research institute by WHOQOL-100 scale. Mod Prev Med. 2008;21:4188-90

31. Wang D, Xu JF. Investigation on quality of life and job stress among nurses of different service length in Dongguan city. Chin J Nurs. 2008;10:936-8.

32. Tang MX, Zhang DM, Liu LJ, Yan XY, Guo Q. Quality of life among soldiers of the first response army in the southeast command area of China. Hosp Adm J Chin People's Liberation Army. 2008;(09):887-90.

33. Tang JR, Zhang XL, Chen YY. The related research of nurse's quality of life and satisfaction with their jobs. Int J Nurs. 2008;(12):1261-3.

34. Dong XX. The research of quality of life and influencing factors of Nanjing and Tianjin's gym full-time gym coachs. master. Suzhou: Soochow University; 2008

35. Liu SH. Study on the quality of life of nurses in the basic level hospital. Nurs Sci. 2008;(07):5-8.

36. Yu HM, Ren XW, Chen Q, Zhao JY, Zhu TJ, Guo ZX. Quality of life of coal dust workers without pneumoconiosis in mainland China. J Occup Health. 2008;50(6):505-11.
37. Zhang LL, Dang WM, Lu L, Shi C, Ma WJ, Yuan LY, et al. An investigation on anxiety status and quality of life of workers in a furniture factory in Beijing. Chin J Ind Hyg Occup Dis. 2008;(10):612-3.

38. Su L. A study of life quality and related factors in jinan middle school teachers. master. Jinan: Shandong University; 2008.

39. Dong WY, Xu JF. Com parison on quality oflifeofnursesin psychiatrichospitaland generalhos pital. Chin J Mod Nurs. 2008;14(16):1758-9.

40. Li XJ, Xie C, Chen F, Huang YX. Controlled study on life quality of doctors in psychiatric hospital and general hospital. Chongqing Med. 2008;37(23): 2727-2728+2731

41. Liu J, Meng DH. Pilot study of reconstruction personnel's quality of life after 5.12 Wenchuan earthquare. J Nanjing Med Univ (Soc Sci). 2009;9(04):342-4.

42. Tang MX. Study on health related quality of life of soldiers in grass-roots army based on population survey. doctor. Shanghai: The Second Military Medical University; 2009

43. Gao WM. A study on the quality of life and it's related factor of hospital nurse in Kunming tertiary hospital. master. Chongqing: Third Military Medical University; 2009

44. Wan CD, Ke LH. Insight of the effect of society supports to the nurse life quality. West China Med J. 2009;24(09):2464-6.

45. Li Z. The study about self-efficacy, quality of life status and its influencing factors of community nurses in Shanxi province. master. Changsha: Central South University; 2009

46. Zhou P, Lu GL, Sun JP, Cui ZH, Gu P. Quality of life and its related factors among employees in CBDs of Changning district, Shanghai city. Chin J Health Educ. 2009;25(5):340-2.

47. Zhang Q. Study on quality of life and affecting factors of nurses in Xin Jiang production and construction group. master. Shihezi: Shihezi University; 2009.

48. Huang Y. Study on the quality of life and health-related behaviors among rural-to-urban migrants in construction sites of Hefei. master. Hefei: Anhui Medical University; 2009.

49. Huang FL, Yu FL, Wang B. Surrey on QOL, psychological health situation in train drivers. Chin J Public Health. 2009;25(06):736-8.

50. Ding JL, Liu AS. Analysis on the quality of life of migrant workers in construction industry and its influencing factors. J Heilongjiang Inst Teach Dev. 2009;28(05):15-7.

51. Song YL. The control study oil relationship between health management changing the lifestyle of female journalists and incidence of proliferative benign breast. master: China Medical University; 2009.

52. Gu Y, Liu Q, Yao JH, Liu JD, Wu JL, Yang YX, et al. Investigation on occupational hazards and quality of life of migrant workers in 3 electronic enterprises in Suzhou city. Shanghai J Prev Med. 2009;21(09):447-448+450

53. Song P, Li JS, Tao SH, Guan WJ. Investigation on the quality of life of slaughter workers in Tangshan city. Chin J Coal Ind Med. 2009;12(11): 1770-1.

54. Liu XL, Liu YJ, Li H, Tian XH. Quality of life in medical workers and its influential factors. J Environ Occup Med. 2009;26(04):399-402.

55. Wang F, Deng B. Quality of life and related factors in middle age intellectuals. Chin J Public Health. 2009;25(10):1259-60.

56. Xing YM. Post-traumatic stress disorder symptoms and its relation with quality of lide in ambulance nurses of Linyi. master. Jinan: Shandong University; 2010.

57. Bai $X$, Zhang C. Research on the life quality of office-bearer in Urumqi. J Xinjiang Med Univ. 2010;33(10):1234-1235+1238.

58. Wang YY, Liu WW, Zhang ZH, Xu XS. Path analysis between work pressure and quality of life among medical professionals in a general hospital. Chin Gen Pract. 2010;13(19):2134-7.

59. Fu HB, Lin XP, Yu HB, Kong DL, Ding YL. Analysis on the quality of life of 260 academic leaders in Guangdong province. Med Innov China. 2010; 07(31):87-9.

60. Liu ZD. Emergency nurses' lifestyle and the relationship between lifestyle and job stress and quality of life. master. Jinan: Shandong University; 2010.

61. Zhang P, Zheng JP, Sun JY, Li RF. A study on quality of life in hot environment workers of steel enterprises and its related factors. J Environ Occup Med. 2010;27(1):46-8.

62. Liu YH. Influence of age and marital status on quality of life of nurses. Chin J Clin Ration Drug Use. 2010:03(11):103-5.

63. Jiang LP. Research on the relationship between the mental health, social support and the quality of life of young peasant workers. master. Fuzhou: Fujian Normal University; 2010. 
64. Tang $J$, Xie JP, Wang LD. Relativity research on mental health condition and survival quality between elementary school teachers and middle school teachers in Tongxiang city. Chin Rural Health Serv Adm. 2010;30(8):687-8.

65. Yao SM, Li W, Song PP, Meng SY, Yu HM. The study of quality of life and related factors among medical college teachers. Mod Prev Med. 2010;37(1): 69-72.

66. Jin LJ, Yang RZ, Lin MJ, Zhou YQ. Effect observation for psychological health education of clinic nurse. J Qiqihar Med Univ. 2011;32(13):2208-9.

67. Xu XP, Zhao H, Hou ZZ, Sheng W. The investigations of quality of life and working pressure conditions of nurses in 3-grade A-class hospitals and community hospitals. Med J Air Force. 2011;27(4):193-5.

68. Lou JF. Evaluation of quality of life and effect of healthy lifestyle intervention for community medical workers. master. Zhanjiang: Guangdong Medical University; 2011

69. Wang QQ, Wang JH. Study on factors affecting the quality of life for nurses working in night shifts. Chin J Ind Hyg Occup Dis. 2011;29(5):353-7.

70. Long SF, Shi X. The study of the quality of life among doctors in three level of first-class hospitals and the relationship between them. Int Med Health Guid News. 2011;17(21):2710-3.

71. Wei LS, Zhou ZH, Zhu CQ. Quality of life and its associated factors in the surface ship officers and men. Hainan Med J. 2011;22(1):13-5.

72. Ye XL, Zhang BZ. Study on the quality of life and mental health as well as their influence factors in armed officers and soldiers from earthquake relief work. Hainan Med J. 2011;22(24):18-20.

73. Wan K, Wang HX, Zhou JX. The life quality of policemen in Kunming and its influencing factors. J Kunming Med Univ. 2011;32(1):128-32.

74. Xiong CD, Chen X, Chen ZM. Correlative analysis of the country hospital performance and quality of life of doctors and nurses in Xianning city. Chin Hosp Manag. 2011;31(03):33-4.

75. Wang WJ, Lin DY. A survey on the quality of life of medical staff. Chin J Health Manag. 2011;05(6):362-3.

76. Zhang R, Li HP, Wang WL. Relationship between quality of life and social support among teachers in medical university. Anhui Med J. 2011;(9):131921.

77. Ma HY, Wu N, Jin B, Li CY, Meng R. Investigation on mental subhealth of some airmen in a navy. J Prev Med Chin People's Liberation Army. 2012 30(01):29-31.

78. $\mathrm{Ma} \mathrm{CH}, \mathrm{Li} X \mathrm{XH}, \mathrm{Wu} Y \mathrm{YH}$, Li DY, Wang JH. Investigation on the quality of life and health-promoting lifestyle of urban migrant workers in Hebei province. J North China Inst Aerosp Eng. 2012;22(05):61-2.

79. Ban YF, Liu CY. On the effect of special education teachers' burnout on their quality of life: the mediating effect of social support. Chin J Spec Educ. 2012;(10):61-6.

80. Wang J, Zhang M, Yang GQ, Yan JH. Influence of violence in the hospital workplace on the psychological status and life quality of nursing staff. Hainan Med J. 2012;23(16):155-6.

81. Hu ZP, Wu CF, Wei NK, Wang Y, Ding SC, Zhang QC. Influencing factors of quality of life among workers in an enameled wire enterprise. Occup Health. 2012:28(18):2180-3

82. Xu JJ. Study on quality of life of senior nurses aged more than 40 years. master. Shantou: Shantou University; 2012.

83. Zhang $H$, Ding $L$, Sun $H$. Correlation between trait emotional intelligence and life quality in medical staff. Chin Gen Pract. 2012;15(31):3657-9.

84. Liu ZM, Bao L, Huang WY, Tan AJ, Lian HQ, Qiu SQ. Investigation and analysis of the qulity of life of migrant workers in electronics factory in Zhuhai. J Front Med. 2012;(31):80-2.

85. Zhang HJ, Dong YP, Liu HC. Quality of life among 354 female migrant workers in Baoding. J Environ Occup Med. 2013;30(7):548-50.

86. Xu XS, Zhang F, Wang YY. Path analysis between work pressure and life quality among nurses in a tertiary hospital in Beijing. Chin J Mod Nurs. 2013;19(6):621-5.

87. Wang YH. Investigation of living quality of employees in public places and influential factors. Anhui J Prev Med. 2013;19(5):350-3.

88. Hu CM, He HM, He L. Investigation of living quality of employees in public places and influential factors. Chin J Public Health. 2013;29(8):1224-7.

89. Tan QZ, Cheng XN, Wu XM. The sleep quality, daytime function and life quality of medical staff with shift work in Panyu district of Guangzhou city. China Mod Doct. 2013;51(8):135-6.

90. Dan PW, Gao S. Investigation on the relationship between smoking and drinking behavior and quality of life among the first-line psychiatric medical staff. Med J Chin People's Health. 2013;25(19):84-6.
91. Wu JB, Hu JF, Chen J, Chen LH, Wei JB. Survey on the living quality of clinical female doctors in level three first-class hospitals, Fuzhou city, 2011. Prev Med Tribune. 2013;19(1):1-2,6.

92. Xing H, Yu W, Chen S, Zhang D, Tan R. Influence of social support on health-related quality of life in new-generation migrant workers in eastern China. Iran J Public Health. 2013:42(8):806-12.

93. Yu JY. Study on the relationship among quality of life, social support and mental health of emergency. master. Changsha: Central South University; 2013.

94. Fu J, Zhang FS. Investigation on the correlation between mental health and quality of life of nurses in Kaifeng district. Med J Chin People's Health. 2013; 25(23):108-10.

95. Zhang XP. Differences of job burnout and quality of life between male and female nurses: a cross sectional study. master. Jinan: Shandong University; 2013

96. Wu CF, Yang YJ, Hu ZP, Ding SC, Zhang QC. Study on the quality of life and its influencing factors among workers in two foundry enterprises of Tongling city. Occup Health. 2013;29(15):1861-5.

97. Geng SX, Liu J, An R, Lv SY, Chai M. Quality of life and coexisting depression and an anxiety of female nurses in general hospitals. Chin J Behav Med Brain Sci. 2013;(02):160-3.

98. Lin LZ, Cheng B, Hu LH, Zhang DQ, Lin PZ, Xiang N. Investigation and analysis of quality of life and influencing factors of female medical workers. J Fujian Med Univ (Soc Sci Ed). 2014;15(04):30-3.

99. He WJ, Cao CG. A correlational study about social support, coping style and quality of life on the new generation of migrant workers. Mod Prev Med. 2014;41(13):2396-2398+2422

100. Li FY, Li YH. Investigation and analysis of influencing factors on quality of life of ICU nurses. China Health Care Nutr. 2014;(6):3560-1.

101. Guo TW, Ma WH, Zhang WY, Pan KX, Zhao JJ, Guo Z, et al. Comparative study on emotion status and quality of life among different occupational groups in Beijing. Occup Health. 2014;30(13):1771-5.

102. Li M, Liu SM, Tian W, Sun LL, Jin P, Luo XL. A study on the quality of life among nurses in hospitals of different grades. Chin Nurs Manag. 2014;(3): 304-305,306.

103. Lao $Y$. The mental health status of human town doctors. master. Changsha: Central South University; 2014.

104. Wang JH, Li ZJ, Dong BY, Wu R, Mu SY. Investigation on the quality of life and its influencing factors of a special forces. People's Mil Surg. 2014;57(9): 956-7.

105. Zhang HX, Zhang XN. Investigation on the quality of life of community nurses. Chin J Sch Doct. 2014;28(1):30-1.

106. Yang Y, Lu XZ. Quality of life and job burnout in kindergarten teachers. Chin Ment Health J. 2014;28(04):298-301.

107. Han YQ, Shi WJ. Investigation on the quality of life of middle shift nurses. J Chin Physician. 2014;(z2):310-1.

108. Wu YQ. The study of correlation between oncology nurses' work environment and quality of life. master. Xinxiang: Xinxiang Medical University; 2014

109. Zhang FX. Study on the relationship among quality of life and mental health of ICU nurses. master. Jinan: Shandong University; 2015.

110. Yang YJ, Guan X, Wang L, Jiao Y, Li Y, Yu F, et al. Analysis on quality of life and its influencing factors among HIV / AIDS prevention and control personnel in Guangxi. Chin J Public Health. 2015;31(5):659-61.

111. Guan $X$, Yang YJ, Wang $L$, Jiao Y, Li Y, Yu F, et al. Quality of life and its influencing factors among HIV / AIDS prevention and control personnel in Heilongjiang province. Chin J Public Health. 2015;31(8):1072-4.

112. Li W. Study on occupational mental health and quality of professional life of nurses. J Med Inf. 2015;(19):196-7.

113. Jiang ZN. The study of the quality of life in rural-to-urban migrants in railwayconstruction sites og Lvliang and health education intervention study. master. Taiyuan: Shanxi Medical University; 2015

114. Miao L, Zhang XN. Study on living quality and influencing factors of nurses in internal medical wards of a grade III-A general hospital. Chin J Sch Doct. 2015;29(2):108-11

115. Tang $\mathrm{H}$. Research on the quality of life and management countermwasures among physicians: a case of a class a tertiary hospital in Guangzhou master. Guangzhou: Southern Medical University; 2015.

116. Kang P, LV Y, Hao L, Tang B, Liu Z, Liu X, et al. Psychological consequences and quality of life among medical rescuers who responded to the 2010 Yushu earthquake: a neglected problem. Psychiatry Res. 2015;230(2):517-23. 
117. Yan JW. Investigation and analysis of quality of life of emergency doctors in Foshan Nanhai District General Hospital. J Med Inf. 2015;(18):341-1.

118. Pan LY, He RB, Liu CH, Yang MF, Luo CX, Wu FJ. Canonical correlation analysis of operating room nurses' quality of life and its influencing factors. Med Equip. 2015;(6):118-120,121.

119. Chen N, Liang F, Liu YC, Liu HR. The impact of occupational stress in sanitation workers on quality of life. J Ningxia Med Univ. 2016:38(11):1261-6.

120. Dai WH, Shi JJ, Zhou CY, Qiu JC, Li LZ, Luo XL, et al. Study on sub health status and related factors of civil service in Nantong city. Chin J Gen Pract. 2016;14(6):1004-6.

121. Hu ZP, Wu CF. Analysis of life quality and its influencing factors among workers in a chemical enterprise. Anhui J Prev Med. 2016;22(3):168-71.

122. Yang HP, Chen GW, Zhang HN. Quality of life of lead-exposed female workers in Shaoguan city and its influencing factors. Pract Prev Med. 2016; 23(7):780-3.

123. Zhao X, Li WN, Gui J, LV H, Liu N, Liu Q, et al. Reseach on current life quality situation of soldiers of the field operations and influence factors. Chin J Mod Nurs. 2016;22(20):2836-40.

124. Tang W, Xiong H. Study on occupational mental health and quality of professional life of nurses. Psychol Doct. 2017;23(21):323-4.

125. Zhang D, Cheng RY, Cai W, Gong GQ, De J. Investigation on quality of life and its influencing factors of the primary level medical staff in Lhasa. China Mod Med. 2017;24(8):157-9.

126. Lai CR, Chen S, Huang JY. Investigation on quality of life of surgical nurses. Mod Diagn Treat. 2017;28(6):1156-7.

127. Zhao GX, Yang XL, Zhao XH. Investigation on quality of life of medical staff in oncology department. Chin Health Qual Manag. 2017;24(1):49-51,58.

128. Xiao J, Huang B, Shen H, Liu X, Zhang J, Zhong Y, et al. Association between social support and health-related quality of life among Chinese seafarers: a cross-sectional study. PLoS One. 2017;12(11):e0187275.

129. Su W. Investigation on nonspecific low back pain and its impact on quality of life among solders of Chinese people's armed police forces of Shanxi province. master. Taiyuan: Shanxi Medical University; 2017.

130. Liu S, Zhang L, Feng D, Zhang Y, Yang J, Wu Y. The quality of life of rural doctors and its affecting factors: a investigation from Macheng city, China. Chin Health Serv Manag. 2017;34(02):134-136+151.

131. Zhang JB, Dong ZQ, Fu YG, Ma ZH. Quality of life and its influencing factors among workers in a mining group, Changzhi city. Mod Prev Med. 2017 44(19):3488-91.

132. Yi YP, Jiang YL, Li YX, Zeng YY, Zhou XD, Jiang $C$, et al. Quality of life and its influencing factors among underground coal miners in Pingdingshan city in 2017. Occup Health. 2018;34(1):17-20,24.

133. Zeng WF, Yan WJ, Song W, Su T. Quality of life and its associated factors in the surface ship officers and men. Acad J Second Mil Univ. 2018;39(2):203-6.

134. Yang XJ, Huang DS, Zhao LJ, Zhang TH, Wan KK. Study on the quality of life among service worker in Yunnan Baoshan. J Med Pest Control. 2018;34(1):34-6.

135. Lu YL, Li XX, Luo BM, Xie J, Wang Y, Zhi XL, et al. Social support and quality of life among new generation of migrant workers in Tianjin city. Occup Health. 2018;34(12):1669-72.

136. Zhao LH. The study about self-compassion and quality of life status and its influencing factors of oncology nurses. master. Tangshan: North China University of Science and Technology; 2018.

137. Xue PP, Wang $H$, Chen $P$, Chen M, Xue YP, Zhou F. Correlation research of clinical nurses' quality of life with sleep quality and job burnout in class III grade A hospitals in Xuzhou. Chin J Mod Nurs. 2018;24(1):17-21.

138. Song J, Zhou LH, Guo Y, Ding L, Wang CY, Sun H, et al. The impact of employee care program on the quality of life of medical staff. Chron Pathematol J. 2018;19(10):1395-7.

139. Yang RJ. Theoretical construction and empirical study on Chinese faculties' occupational mental health. doctor. Xi'an: Shaanxi Normal University; 2018

140. Yu Q, Jiang YZ. Effects of occupational mental health on quality of life of nurses. Guide China Med. 2019;17(35):298-99.

141. He HW. Study on the correlation between occupational mental health and life quality of nurses. Health Guide. 2019;(18):357.

142. Song L. Analysis of psychological health and quality of life of female nurses in a hospital in shenzhen and its influencing factors. master. Shenyang: China Medical University; 2019

143. Ma KL, Wang H, Gao X, Huang JJ, Sun CM, Qiao N, et al. Sleep quality mediating the association of personality traits and quality of life among underground workers and surface workers of Chinese coal mine: a multi-group SEM with latent response variable mediation analysis. Psychiatry Res. 2019;272:196-205.
144. Asante JO, Li MJ, Liao J, Huang YX, Hao YT. The relationship between psychosocial risk factors, burnout and quality of life among primary healthcare workers in rural Guangdong province: a cross-sectional study. BMC Health Serv Res. 2019;19(1):447.

145. Zhu RG. The correlation between professional identity, quality of life and job satisfaction of male nurses in Qingdao. master. Qingdao: Qingdao University; 2019

146. Wu Y, Li S, Yang J. Moderating role of perceived social support in the relationship between emotion regulation and quality of life in Chinese ocean-going fishermen. Front Psychol. 2020;11:954.

147. Zeng LN, Lok Kl, An FR, Lu L, Jackson T, Ungvari GS, et al. The prevalence of burnout and its associations with demographic correlates and quality of life among psychiatric nurses in China. Psychiatr Q. 2020.

148. Liu WM, Ma J. Research on the impact of working pressure on psychological stress and life quality of orthopedics nursing staff. China Med Pharm. 2020;10(18):243-5.

149. Luo GC, Yu ZZ, Chen YC, Cai JF, Liu Y. Quality of life among white-collar workers in Yinzhou district of Ningbo city. Chin Rural Health Serv Adm. 2020;40(01):65-8.

150. Wang K, Qin XY, Feng YC, Li JY, Xiao L, He JW, et al. Survey on sleep quality and life quality of navy servicemen stationed on an island. Acad J Second Mil Univ. 2020;41(2):211-5.

151. Wei WH, Song XQ, Guo M. Effect of occupational stress on quality of life of pediatricians and pediatric nurses in Zhengzhou. Med Soc. 2020;33(4):118-21.

152. Chen CQ, Chen GW, Yang HP. Study on the quality of life of radiation workers in Shaoguan city. Chin J Urban Rural Enterp Hyg. 2021;36(01):23-7.

153. Skevington SM, Lotfy M, O'Connell KA. The World Health Organization's WHOQOL-BREF quality of life assessment: psychometric properties and results of the international field trial. A report from the WHOQOL group. Qual Life Res. 2004;13(2):299-310.

154. Deng Q, Wang LM, Zhang M. Quality of life and related influencing factors in Chinese adults. Chin J Epidemiol. 2016:37(02):243-7.

155. Chen ML, Chen CJ, Yeh WY, Huang JW, Mao IF. Heat stress evaluation and worker fatigue in a steel plant. AlHA J (Fairfax, Va). 2003:64(3):352-9.

156. Xiang J, Bi P, Pisaniello D, Hansen A. Health impacts of workplace heat exposure: an epidemiological review. Ind Health. 2014:52(2):91-101.

157. Boschman JS, van der Molen HF, Sluiter JK, Frings-Dresen MH. Musculoskeletal disorders among construction workers: a one-year followup study. BMC Musculoskelet Disord. 2012;13(1):196.

158. Park H, Park HD, Jang JK. Exposure characteristics of construction painters to organic solvents. Saf Health Work. 2016;7(1):63-71.

159. Ministry of Housing and Urban-Rural Development of People's Republic of China. Notice on production safety accidents of housing Municipal Engineering. Available from: http://www.mohurd.gov.cn/index.html. Accessed 5 Jun 2021.

160. Mokarami H, Stallones L, Nazifi M, Taghavi SM. The role of psychosocial and physical work-related factors on the health-related quality of life of Iranian industrial workers. Work. 2016:55(2):441-52.

161. Leyshon R, Chalova K, Gerson L, Savtchenko A, Zakrzewski R, Howie A, et al. Ergonomic interventions for office workers with musculoskeletal disorders: a systematic review. Work. 2010;35(3):335-48.

162. Barbieri DF, Nogueira HC, Bergamin LJ, Oliveira AB. Physical and psychosocial indicators among office workers from public sector with and without musculoskeletal symptoms. Work. 2012;41(Suppl 1):2461-6.

163. Elshaer N. Prevalence and associated factors related to arm, neck and shoulder complaints in a selected sample of computer office workers. J Egypt Public Health Assoc. 2017;92(4):203-11.

164. Wang $L$, Chang C, Ding HF, Jiang SL, Wei YF. Life quality of MSWs and the related factors in Beijing. Chin J AIDS STD. 2018;24(8):796-9.

Ready to submit your research? Choose BMC and benefit from:

- fast, convenient online submission

- thorough peer review by experienced researchers in your field

- rapid publication on acceptance

- support for research data, including large and complex data types

- gold Open Access which fosters wider collaboration and increased citations

- maximum visibility for your research: over $100 \mathrm{M}$ website views per year

At BMC, research is always in progress.

Learn more biomedcentral.com/submissions 